\title{
DER BLUFF
}

\section{Die Institution Universität in ihrer Wirkung auf die Arbeitsweise ung das Bewußtsein ihrer Mitglieder}

\section{Wolf Wagner}

\section{Vorbemerkung}

Schon vom Titel her scheint der folgende Aufsatz mit dem redaktionellen Korrz der „Probleme des Klassenkampfs" schwer vereinbar, scheint aus dem Rahme. zu fallen. Bedenkt man jedoch, daß die meisten Autoren und Leser dieser Zeitschrift entweder selbst Studenten und Dozenten oder durch die universitäre Ausbildung stark geprägt sind, dann erscheint es schon nicht mehr so abwegig, hier eine Reflexion auf die eigene Situation anzustellen, erst recht, wenn man es sonst für möglich halten sollte, daß eine solche Zeitschrift auf die Dauer den Eindruck einer Art Weltgeistes erwecken müßte, der alles in sich reflektiert, die Basis seiner eigenen Existenz aber nicht zu erkennen gibt.

Der Aufsatz ist im Rahmen eines Ausschusses der SAZ (Sozialistische Assistentenzelle am Otto Suhr Institut) über "Didaktik des Hochschulunterrichts“ entstanden. In diesem Rahmen sollte er für die Universität das leisten, was Peter Fürstenaus Aufsatz „Zur Psychoanalyse der Schule als Institution“ 1) für den Bereich der Schule unternimmt, nämlich herauszuarbeiten, in welcher Weise das Verhalten der Studenten zueinander und das Verhältnis zwischen Dozenten und Studenten durch die Spezifik der Institution Universität auf einer unbewußten Ebene vorgeprägt ist. Erst wenn eine solche Prägung bewußt gemacht ist, wenn also bekannt ist, welche Einstellungen und Verhaltensweisen ganz spezifisch durch die Institution Universität gefördert oder behindert werden, ist es vielleicht möglich, daß inhaltlich-politische Zielsetzung und Durchführung der Lehrveranstaltungen nicht ständig unkontrollierbar auseinanderfallen und die Studenten dadurch in einer Tendenz zu vereinzelter Passivität oder theoriefeindlichem Aktivismus bestärkt werden; ist es vielleicht möglich, daß stattdessen unsere Lehrveranstaltungen vielleicht eher zu einem politischen Lernzusammenhang solidarisch agierender Studentengruppen werden können.

Bei der Bearbeitung dieses noch bloß didaktischen Problems erweiterte sich aber die Fragestellung ganz folgerichtig: Wenn es möglich sein sollte, die spezifische Prägung 2) durch die Universität ganz allgemein darzustellen, so müßte es auch

1) In: Das Argument, 29, 6. Jg., H. 2, Mai 1964, S. 65-78.

2) Wenn hier von der ,spezifischen" Prägung durch die Universität gesprochen wird, so ergibt sich eine methodische Schwierigkeit, auf die Peter Fürstenau in:,Über Beratung, Therapie und Erforschung sozial definierter Neurotikergruppen", in:Psychische Störungen bei Studenten - Symposion vom 22.-24.3. 1968 in Berlin, Hrsg. H.U. Ziolko, Stuttgart 1969, S. 86 und 37 hinweist: Alle Aussagen über die spezifische Situation von Studenten als Studenten müssen sich auf Plausibilitätsüberlegungen beschränken, da es zwar umfangreiches Datenmaterial über studentisches Verhalten und Einstellungen gibt, 
möglich sein, zu untersuchen wie sich die Wirkung der Institution Universität auf ingre Mitglieder wandelt, wenn sich die allgemeinen Rahmenbedingungen verändern.

Oder anders ausgedrückt: Die Untersuchung der allgemeinen, überindividuelien Verhaltensweisen und Einstellungen universitärer Intellektueller ist die Untersuchung des ,subjektiven Faktors" auf der Ebene der universitären Intelligenz. Eine solche Untersuchung muß daher an sich selbst den Anspruch stellen, aufzeigen zu können, welche Bedingungen gegeben sein müssen, damit sich das Bewußtsein der universitären Intelligenz nicht nur punktuelle und individuell, sondern massenhaft wandeln kann, von den Verbindungsstudenten und existentialistischen Kulturkritikem der fünfziger Jahre zu den - zumindesî der Intention nach - solidarisch agierenden Studentengruppen der antiautoritären Studentenrevolte der sechziger Jahre. Natürlich wäre es notwendig, dazu die ganzen bildungs-ökonomischen Ergebnisse in ihrem Zusammenhang mit der Entwicklung der BRD zu einem Erklärungsversuch zusammenzufassen. Das könnte hier aber höchstens in Form eines Bluffs geleistet werden. Es geht hier daher also vielmehr nur darum, auf einleuchtende Weise darzustellen, wodurch das Bewußtsein der universitären Intelligenz besonders geprägt sein wird und wie diese besondere Prägung zum Verlauf der Studentenrevolte und ihrer weiteren Entwicklung beigetragen haben mag. Um Mißverständnisse zu vermeiden muß schon hier gesagt werden: der Ansatz dieses Aufsatzes ist nur ein Moment in der Enklärung der Studentenrevolte und ist sicherlich stark geprägt durch meine eigenen Eriebnisse während der Studentenbewegung, die jemandem, der damals z. B. im SDS selbst bei der Vorbereitung von Aktionen und Veranstaltungen beteiligt war, sehr fremd erscheinen müssen. Doch ist diese Sichtweise nicht bloß subjektiv einzelne Erfahrung, sondern wird von vielen geteilt, die damals auch ihre entscheidenden Lernprozesse in den autonomen Gruppen an der ,Basis ${ }^{6}$ mitgemacht haben.

Der Aufsatz gliedert sich in zwei Teile, einen abstrakt-allgemeinen Teil, in dem versucht wird herauszuarbeiten, welche Einstellungen und Verhaliensweisen notwendig sind, um sich als Universitätsmitglied - als Dozent oder Student 3) halten zu können, und einen konkret-handgreiflichen, in dem die besonderen uni-

aber keinerlei empirisches Material vorliegt über nichtstudentische, gleichaitrige Mittelklasse-Jugendliche. Dies wäre aber notwendig um die Behauptung einer spezifischen Prägung durch die Universität empirisch zu untermauern. Das empirische Material, das im weiteren angeführt wird, kann daher keine Beweiskraft haben, sondern soll lediglich die Plausibilität der Erklärungsversuche stützen, und dem Verdrängungsversuch entgegenwirken, die hier behandelten Probleme als bloß OSI-spezifisch abzutun.

3) In diesem Teil werden die ,, anderen Dienstkräfte" Beamten der 'Universität, die nicht Dozenten sind, noch aus der Analy se ausgespart, da sich für sie diese Frage im allgemeinen nicht anders beantwortet als für andere vom Staat beschäftigte Lohnabhängige, d. h. die Antwort ist einerseits wesentlich bestimmt durch die besonderen Tätigkeitsmerkmale, ob als Eisenbahner, pförtner oder Bibliotheksange. stellte oder Sekretärin, und andererseits durch das „,Treueverhältnis“ zum Staat und die besondere persönliche Abhängigkeit vom Vorgesetzten. Die besondere Lage der ,anderen Dienstkräfte"s wird daher besser im Zusammenhang mit den Verhaltensweisen der Studenten und Dozenten ihnen gegenüber behandelt, also im zweiten, dem konkreteren Teil. 
versitären Verkehrsformen für die unterschiedlichen Gruppen analysiert werden.

Es wurde mehrfach erwogen, diesen ersten, abstrakteren Teil wegzulassen, da er manche Leser davon abhalten könnte, den wichtigeren zweiten Teil zu lesen. Dann könnte die Analyse im zweiten Teil aber zu leicht als spritzig feuilletonis̈tische, Oberflächenbetrachtung " abgetan werden. Erst wenn gezeigt worden ist, daß sich die besonderen universitären Verkehrsformen mit Notwendigkeit aus der Rolle ergeben, die der Universität im Reproduktionsprozeß der kapitalistischen Gesellschaft zufällt, kann der Versuch, sich die universitären Zwänge bewußt zu machen und sich gegen sie zu wehren, nicht mehr mit der Phrase von der ,kleinbürgerlichen Borniertheit" abgewehrt werden. Es muß klar werden, daß eben dieser Versuch ein notwendiger Beitrag zu einer Strategie-Bestimmung an der Universität bedeuten muß, da er an denjenigen Bedürfnissen der Universitätsmitglieder anzuknüpfen versucht, die im Widerspruch zur kapitalistischen Funktionsbestimmung der Universität stehen 4).

Auf keinen Fall darf man den ersten Teil als Versuch zur Klassenanalyse der Studenten mifverstehen. Hier geht es vielmehr darum: mit dem Übergang der antiautoritären Studentenrevolte in die ML-Bewegung wurde das an der eigenen Bedurfnisstruktur, auf die eigene Emanzipation orientierte Bewußtsein als ,kleinbürgerliches' an einem, proletarischen ${ }^{6}$ Bewußtsein normativ gemessen und zusarnmen mit den Ansätzen der Studentenrevolte - eben nicht aufgenommen und über seine Beschränkung hinausgefiuhrt, sondern pauschal als bedeutungslos oder gar hinderlich bei ,der Arbeit im Proletariat' abgetan 5).

Die Frage ist nun: ist das Bewußtsein der Universitätsmitglieder - insbesondere der Studdenten - tatsächlich dadurch geprägt, daß sie Kleinbürger sind im ökonomischen Sinne, also einfache Warenproduzenten, die zwar Produktionsmittel besitzen (ind vielleicht - als Professoren - einige Lohnarbeiter beschäftigen), aber doch selbst mitarbeiten müssen? Kann man alle Aussagen, die Marx, Engels oder Lenin über de Kleinbuirger gemacht haben, von daher einfach bruchlos auch auf die universitart inteligenz übertragen 6)? Oder besteht das Richtige in der Rede von der wembivenhohen Intelligenz" nicht vielmehr in einer strukturellen Analogie, die zwar âmliche, aber durchaus auch andere und selbständige Bewußtseins- und

4) Gi Ganheimer Papier: ,Wie können wir die psychische Selbstbefreiung systematisch in masere politische Praxis aufnehmen - in welcher Beziehung steht die psychische selbstbefreiung zur politischen Schlagkraft"; in allen linken Buchläden erhältlich, Broschure hektographiert.

5) Besonders beispielhaft in: „Vorläufige Plattform der Aufbauorganisation für die Kommunistische Partei Deutschlands" (Rote Presse Korrespondenz, Nr. 56/57, 2. Jg. 1970): ,Zweitens gibt es sogenannte Arbeitsschwierigkeiten bei der Abfassung von Papieren, Flugblättern usw. Die Methode der Berichtigung ist: Man muß erstens erklären, dalb diese sog. Arbeitsschwierigkeiten in einer bestimmten Phase der Studentenbewegung in Mode waren und mufs die dahinterstehende Ideologie bekämpfen." (S. 7) „Diese Verhaltensweisen haben ihren gesellschaftlichen Ursprung in der kleinbürgerlichen und bürgerlichen Ideologie; das ist bei der Erziehungsarbeit zu beachten." (S. 8).

6) So: Monika Steffen und Klaus Funken „Die einfache Kaderproduktion. Thesen zur Studentenbewegung als kleinbürgerlicher Massenbewegung" in: Kursbuch 25, Oktober 1971, S. $.23-141$. 
Verkehrsformen begründen kann als bei den echten, ökonomisch bestimmten Kleinbürgern? Diese Fragestellung - als Ergebnis der Geschichte der Studentenbewegung - gibt das Erkenntnis-leitende Interesse des ersten Teils an.

Im Übrigen ist es wahrscheinlich am besten, wenn man zuerst ein wenig im zweiten Teil (möglichst weit hinten) herumliest, um zu sehen, worauf das ganze hinauswill - dann sollte man aber nochmal von vorn anfangen und der Logik der Gedankenführung folgen.

\section{Teil I: Der universitäre Produktionsprozeß}

\section{A. Die wich tigsten Fragen}

Eine Untersuchung der allgemeinen Bewußtseinslage der universitären Intelligenz im Kapitalismus muß unserer Vorstellung nach so vorgehen: zuerst muß die besondere Weise der Produktion dargestellt werden, der sie unterwofen ist, und welchen konkreten Beitrag diese zur materiellen Produktion und Reproduktion des gesellschaftlichen Lebenszusammenhanges unter kapitalistischen Bedingungen leistet. Damit ist seine allgemeine gesellschaftliche Bedeutung umrissen und zugleich ist die Analyse des konkreten Arbeitsprozesses in seiner Funktion als bestimmte Bedürfnisse befriedigende Arbeit eingeleitet. Diese Seite ist darum von besonderer Wichtigkeit, weil sie die konkrete Form der Auseinandersetzung mit der gegebenen gesellschaftlichen und natürlichen Umwelt ausmacht und damit ganz entscheidend den Bereich möglicher Erfahrungen eingrenzt. Aber diese konkrete Seite ist bekanntlich nur eine Seite eines jeden Produktionsprozesses in der entwickelten kapitalistischen Gesellschaft.

Da die gesellschaftliche Reproduktion in der kapitalistischen Produktionsweise durch den Austausch von Wertäquivalenten in der Zirkulation vermittelt ist, muß sich jeder Produktionsprozeß - auch der universitäre - auf diese Wertvermittlung hin orientieren. Denn die Stellung in diesem Wertvermittlungsprozeß entscheidet für den Einzelnen wie für die unterschiedlichen universitären Gruppen, wie und wieviel sie vom gesellschaftlichen Gesamtprodukt zu ihrer eigenen materiellen Reproduktion erhalten.

Beide Seiten zusammengenommen bilden die Grundlage, auf der folgende Überlegungen angestellt werden können: diejenigen Einstellungen und Verhalsensdispositionen, die die individuelle materielle Reproduktion in diesem Bereich auf besserer Basis sichert, werden - weil sie größere Sicherheit bieten, in dem besonderen Bereich zu verbleiben - mit größerer Wahrscheinlichkeit anzutreffen sein. Diese erhöhte Wahrscheinlichkeit bestimmter Einstellungen und Verhaltensdispositionen war'gemeint, als vorhin von der unbewußten Vorprägung durch die Institution Universität gesprochen wurde. Es wird eine Unmenge anderer Einstellungen und Verhaltensdispositionen geben, die man an der Universität antreffen kann, die aber nicht spezifisch durch die Institution Universität gefördert werden, die also für eine Untersuchung der universitären Bewußtseinsformen irrelevant sind.

Die Fragen, die Aufschluß geben können über die besonderen Bewußtseins- 
formen der universitären Intelligenz, lauten also 7):

1. Welche Einstellungen und Verhaltensweisen werden durch die besondere Form der konkreten, gebrauchsorientierten Arbeit an der Universität als Voraussetzungen einer einigermaßen kontinuierlichen Arbeit in dieser Institution als Minimum gefordert, bzw. besonders gefö rdert oder unterdrückt?

2. Welche Einstellungen und Verhaltensdispositionen werden durch die Institution Universität als Mittel zur Erlangung eines persönlichen Eiakommens befördert bzw. unterdrückt?

(Diese Fragen sollen im zweiten Teil im einzehen beantwortet werden, wenn in den nun folgenden Überlegungen zur Stellung der Universität im gesellschaftlichen Reproduktionsprozeß die notwendigen Voraussetzungen dazu geschaffen sind.)

\section{B. Überlegungen zur Stellung der Universität im gesellschaftichen Reproduktions- prozeß}

Diese Überlegungen müssen separat nach dem universitären Produktionsprozelß als Forschungs- und als Ausbildungsprozeß, bei letzterem wieder separat nach der Stellung von Dozenten und Studenten gegliedert werden.

\section{Der Forschungsprozeß}

1.1. Das besondere Produkt des universitären Produktionsprozesses als Forschungsprozeß ist Wissen als Lösung von Problemen.

1.2. Die Probleme, die gelöst werden, kann man nach dem Grad ihrer unmittelbaren Relevanz für den materiellen Produktions- und Reproduktionsprozeß des gesellschaftlichen Lebenszusammenhanges unter kapitalistischen Bedingungen unterscheiden:

a) Von unmittelbarster Relevanz sind die technischen Wissenschaften und die Resultate der ,angewandten ' Forschung in den Naturwissenschaften, deren Ergebnisse nach Rentabilitätsgesichtspunkten ausgewählt in den Verwertungsprozeß des Kapitals eingehen und individuellen Kapitalen zu Extramehrwert verhelfen können, also ein relativ unvermittelter Faktor in der Beeinflußung der Produktivkraft der Arbeit darstellen. Über die tatsächliche Anwendung der ,angewandten' Wissenschaften im kapitalistisch betriebenen Reproduktionsprozeß entscheidet das Kriterium der Profitsteigenung und -sicherung, das auch die Problemstellungen und die Entwicklung in diesen Wissenschaftsgebieten entscheidend prägt. Auch von der stofflich-konkreten Seite her sind sie von allen Wissenschaften von unmittelbarster Relevanz für den materiellen Reproduktionsprozeß, wenn sie dazu beitragen, z. B. die Versorgung der Bevölkerung auf besserem Niveau oder die medizinische

7) Fürstenau formuliert das Problem se: ,Aufgabe... ist, die soziale Strukiur und Funktionsweise - in unserem Fall: der Hochschule - in Richtung darauf zu klären, ob überhaupt und gegebenenfalls welche spezifischen, selektiven Einflüsse von der Hochschule als Institution auf die Persönlichkeitsstruktur ihrer studentischen Mitglieder regelhaft einwirken und wie die Studenten diese Einflüsse psychisch in der Regel verarbeiten." (Fürstenau; Über Beratung..., a. a. O.; S. 85) Seine Antworten bleiben in dem Aufsatz jedoch leider auf den Aspekt der Beratertätigkeit in Gruppen psychisch gestörter Studenten beschränkt. 
Eehandung effektwer zu garmieren. Aber whoh hier ist die Wirkung vermittelk uber die auf Verselbständigung drängende Wenseite: bessere Versorgung und effektiveres Gesundheitswesen werden nur zu leicht zw Basis verschärfter Ausbeutung an Arbeitsplatz.

b) Schon vermittelter, aber immer noch von unmitebar einleuchtender Bedeutung ist der Bereich der naturwissenschaflichen und metheratischen

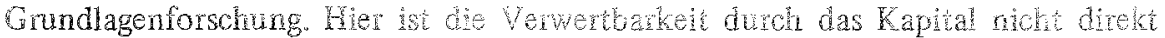
gegeben, sonden erse, went de theretisenen Problemboungen techisch anwon bar geworden sind - abso uber de Sufe der Kategone a) gelaufen sind. Fir ds stofflich-konkrete Reproduktion sind die problemlösungen meist unmittelbare relevant, da sich die Probleme oft selbst aus den Schwierigkeiten der materielien Poduktion und Reproduktion des geselgohticher Lebensusamenhanges satlen, z. B. die Krebsionschung, die Okologe oder genetische Forschngen. Bei ener großen Zahl von Problenstehngen ist de gesellschaftliche Relevanz aber nicht unmittelbar einsichtig. In desen Fallen lavet die Vemithung zur gesellschaftichen Relevanz uber zwei Strănge: eirral uber de Gewähung und Verweigenung von finanziellen Mitteln, zun anderer wber die Enwickhng der Wissenschaftsgeschichte, d. h. dariber daf bestiminte Poblenstellungen wissenschaftsimmanent an mo heren Punkten in unterschiedichen Zusammenhängen als Voraussetzung zur Lösung anderer Probleme aúftauchen oner dab bertmmet Fragenkonplexe allgemen als Liicken oder Widerspruche in der algeneinen Theorie uber den Gegenstand bekani sind, z. B.,die Elementarteilchenforschung, die Frage nach einer mehrwertigen 1 ow gik oder astronomische Forschungen ther den Ursprung der Pulsare. Daneben gibt es aber auch auf dieser Ebene Picblemstelingen, deren Entstehen bestenfalls indwdualpsychologisch enklarbar scheint, de abe trotadem nicht behindert werden, as sie möglicherweise doch geselischaftich relevante Ergebnisse bringen konnten.

c) Der größte Teil der aligunein wblehen Fragestellungen in den Forschuss. bereichen der Sozialwissenschanten, peohts. W. Wrischaftswissenschaften sind woht von direkter Relevanz fur dis stofflchmaterielle gesellschaftiche Reproduktion. Thre Relevanz liegt viemehr dam, die keprodukton der besonderen historischin Form des geseilschaftichen Zasammenhanges ideologisch zu sichern. Dazu brazchen sich die an den Forschungprozeson beteingten Wissenschafter gar nicht bewußt das $\mathbb{Z}$ iel zu setzen, der Aurecherhahng der Herschaft des Kaptals

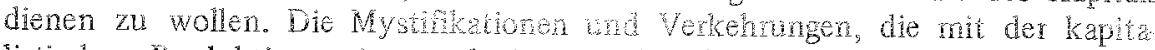
Iistischen Produktionsweise produzien werden (etwa die Fetischisierung gesel schaftlicher Venältnisse in Bezterungen zwischen Dingen) lassen Theorien we die Produktionsfaktorentheorie und die ing entsprechende Pluralismustheorie als Resultate emsthafter und durchaus kitischer wissenschafticher Anstrengung enistehen. Da so alle Produktionsfaktoren gleichmaßig an der Enstellung der knappen, Resourcen" betailigt schemen, könen sich diese Wissenschaftsgebiete darauf beschränken, den geselschafhichen butzen' ihres Einsatzes zu optimieren oder aber sich ganz der Aralyse von partikularisierten Spezialgebieten widnen, ohne nach der Einbetung in den gesellschatichen Gesamtzusammenhang fragen zu müsen. Beides befestigt die Mystifikationen und Verkehrungen und hat so objektiv 
ideologischen Charakter 8).

d) Schließlich gibt es noch die Gebiete der philosophischen Fakultät: Germanistik, Philosophie etc., bei denen der Zusammenhang zur materiellen Reproduktion des gesellschaftlichen Lebenszusammenhanges in seiner besonderen historischen Form sehr vermittelt ist: während bei den technischen Wissenschaften der konkret-stoffliche Gegenstand als der eigentliche Stoff erscheint, mit dem man umgeht, und sich die Anforderungen des Verwertungsprozeßes als Rentabilitätsforderungen zu diesem Stoff äußerlich zu verhalten scheinen und als von außen kommende Anforderungen deutlich erkennbar sind, scheinen sich die philologischen und philosophischen Wissenschaften unbeeinflußt von der kapitalistischen Form der Gesellschaft mit einem sehr flexiblen, beliebig formbaren Stoff zu beschärfigen, nämlich mit Inhalt und Form menschlicher Reflektionen. Diese Indifferenz gegenüber der besonderen historischen Form der Gesellschaft ist aber in doppelter Weise eine Illusion: einmal sind diese Reflektionen in ihren Denkformen durch die Entwicklung der Waren-produzierenden Gesellschaft geprägt, zum anderen unterliegen sie in ihrem Inhalt meist denselben Mystifikationen und Verkehrungen wie die Wirtschafts und Sozialwissenschaften nur eben in noch vermittelterer und noch schwerer erkennbarer Weise (z. B. die Vorstellung vom moralischen Individuum) 9). Wenn auch viele dieser Reflektionen den Keim dazu in sich tragen, den Charakter der kapitalistischen Gesellschaft treffen und ihre Auswirkungen kritisieren zu können, weil ihr gesellschaftlicher Gebrauchswert eben darin liegt, die chaotischen Lebenserscheinungen sinnhaft $\mathrm{zu}$ interpretieren und so of genug das Leben erst lebenswert zu machen, so haben sie doch meist objektiv die Funktion, die kapitalistische Gesellschaft als besondere Form der gesellschaftIichen Reproduktion ideologisch zu sichern. (Dies wird richtig wirksam eigentlich erst dadurch, daß in diesen Bereichen die Ausbildung der zukünftigen Ausbilder schwerpunkimäßig lokalisiert ist und über diesen Umweg die Reflektionen zur bürgerlichen Ideologie vereinfacht als gesellschaftlich herrschendes ,Gedankengut verbreitet werden.)

Diese Wissenschaftsbereiche sind aber doch so weit von den Erfordernissen des materieilen Reproduktionsprozesses und den Verwertungszwängen entfernt, daß hier häufiger als anderswo Verselbständigungen sich entwickeln und For-

8) Dazu für Interessierte folgende weiterführende Literatur, die ich selbst nicht gelesen habe, die mir aber für diesen Zusammenhang empfohlen worden ist: Beiträge zur maxistischen Erkenntnistheorie; Hrsg.: Alfred Schmidt; (es 349) Frankfurt/M 1969.

Helmut Reichelt: Zur logischen Struktur des Kapitalbegriffes bei Karl Marx; Frankfurt/M 1969.

Hans-Jürgen Krahl: Konstitution und Klassenkampf; (Neue Kritik) Frankfurt/M 1971.

9) Auch folgende Hinweise auf weiterfuhrende Literatur kann ich nur weitergeben, da ich sie selbst nicht gelesen habe:

Alfred Sohn-Rethel: Geistige und körperliche Arbeit; (Suhrkamp) Frankfurt/M 1970. ders.: Materialistische Erkenntniskritik und Vergesellschaftung der Arbeit; Internationale marxistische Diskussion 19; (Merve) Berlin 1971.

Karl Marx: Zur Kritik der Hegelschen Rechtsphilosophie; MEW 1, S. 378-391; (Dictz) Berlin.

Kritik der bürgerlichen Germanistík: Das Argument 49;10. Jg. Dezember 1968, Hett 6. 
schungszweige entstehen, deren Existenz nur noch mit den im Teil II beschriebenen Mechanismen erklärt werden kann.

Aber ob gesellschaftlich relevant oder nicht: Jedes Wissen, das ein Problem löst, befriedigt menschliche Bedürfnisse irgendeiner Art - hier das, Antwort auf eine Frage zu bekommen. Eine jede solche Antwort hat also Gebrauchswert.

1.3. Der Produktionsprozeß aller Arten von Wissen hat im Kapitalismus folgenden äußeren Verlauf und folgende Voraussetzungen:

a) Es müssen ausgebildete Wissenschaftler da sein, die genügend Zeit aufwenden und genügende Qualifikation ausweisen können, um das Problem zu lösen. D. h. Personen müssen für die Zeit der Problembearbeitung selbst und für die Zeit der vorgängigen Qualifikationen von anderen gesellschaftlichen Arbeiten freigestellt und solange durch das Produkt der Arbeit anderer versorgt werden. Im Kapitalismus geschieht dies durch extreme und dauerhafte Arbeitsteilung, durch Trennung der geistigen und physischen Potenzen des Produktions- und Reproduktionsprozesses. Da es keine gesellschaftliche Planung gibt, kommen die Wissenschaftler an das für ihre eigene Reproduktion notwendige Produkt der Arbeit anderer nur über den Weg des Äquivalententausches in der Zirkulation. Sie müssen sich zu ihrem Produkt - egal ob es Ware ist oder nicht - verhalten wie zu einer tauschbaren Ware, für die sie Wertäquivalente erhalten oder aber sich selbst als Lohnabhängige begreifen, die ihre Ware Arbeitskraft verkaufen.

b) Das Problem muß sich überhaupt stellen können und muß vom Wissenschaftler erkennbar sein.

Da im Kapitalismus die materielle Produktion und Reproduktion des gesellschaftlichen Lebenszusammenhanges vom Verwertungsstreben der Einzelkapitale bestimmt ist, stellen sich die meisten Probleme, die eigentlich Probleme der gesamtgesellschaftlichen Reproduktion sind, allein in der Form von Verwertungsschwierigkeiten dieser Kapitale, z. B. als unterdurchschnittliche Profitrate wegen überhöhter Kosten. Gelingt es nun einem einzelnen Kapitalisten, eine Lösung des Problems zu finden, also z. B. durch eine Erfindung, den Kostenpreis seiner Waren radikal zu senken, so ist für ihn nicht nur ein wissenschaftliches Problem gelöst, sondern er kann einen Extraprofit realisieren. Dies kann er aber nur solange wie er der einzige ist, der über die Problemlösung verfügt. Er wird also alles tun müssen, um das Wissen geheim, d. h. privat zu halten. Deshalb ist er durchaus bereit, für den privaten Besitz, d. h. das ausschließliche Nutzungsrecht an diesem Wissen, einen Preis zu zahlen. Das Wissen wird gegen Geld eingetauscht. Es ist Ware geworden. Es ist aber nur Ware geworden, weil der Markt, auf dem es verkauft wird, radikal beschränkt worden ist - weil es aus einem allgemein zugänglichen Gebrauchswert zum Gegenstand der einmaligen Konsumtion durch den Käufer gemacht worden ist, die auch dem Produzenten des Wissens den gesellschaftlich relevanten Gebrauch dieses Wissens in einem anderen als diesem einmaligen Verkaufszusammenhang verbietet. Wäre nämlich die Problemlösung weiterhin allgemein zugänglich und verwendbar, würde kein Kapitalist für den Kauf der Problemlösung etwas bezahlen, genausowenig wie er bereit ist, Geld für den Kauf von Atemluft aufzuwenden.

Andererseits ist jedoch unabdingbare Voraussetzung der Produktion von Wissen als Lösung konkreter Probleme, sogar Voraussetzung dafür, daß sich viele 
Probleme überhaupt stellen können: Informationen über den Gegenstand, und zwar möglichst vollständige und für Wissenschaftler allgemein zugängliche und nutzbare Informationen. Jede Einschränkung des Zugangs und der freien Verwendung von problemrelevanten Informationen erhöht die Wahrscheinlichkeit einer falschen Fragestellung und erst recht die Wahrscheinlichkeit falscher Problemlösungen selbst bei richtiger Fragestellung.

Zudem müssen Wissenschaftler auch während des Problemlösungsverfahrens mit anderen Wissenschaftlern in Gedankenaustausch stehen, um Lösungsansätze zu überprïfen und um die endgültige Lösung sich bewähren lassen zu können. Soll der Wissenschaftsbetrieb insgesamt kontinuierlich weitergehen, so darf die Lösung des Problems nicht unveröffentlicht bleiben, denn sie ist nichts anderes als gesuchte und nun gefundene Information zur Lösung weiterer Probleme: sie muß von ihrem Entdecker und von anderen Wissenschaftlern weiterverwendet werden können. Jeder Verschluß von neuen Problemlösungen behindert die Entwicklung der Wissenschaft als konkret nützliche Arbeit zur Lösung konkreter Probleme.

Dadurch, daß Wissen nur dann zur Ware und ,,verwertet" werden kann, wenn es privatisiert wird, treten die Bedingungen, die den Produktionsprozess des Wissens zum Wertbildungsprozess machen können, in direkten und diametralen Widerspruch zu den Bedingungen seiner Produktion im Produktionsprozess als konkretem, gebrauchswertorientiertem Arbeitsprozeß.

Für den gebrauchswertorientierten Arbeitsprozeß haben wir als Bedingung der Möglichkeit richtiger und gesellschaftlich relevanter Problemlösungen die allgemeine Zugänglichkeit und Nutzbarkeit aller Informationen - insbesondere auch der neu produzierten Problemlösungen - herausgearbeitet. Zur Ware können Problemlösungen aber nur werden, wenn sie diese allgemeine Zugänglichkeit und Nutzbarkeit verhindern, wenn sie also die Bedingungen der Möglichkeit ihrer eigenen zukünftigen Produktion beschränken.

Die völlige Monopolisierung von Problemlösungen im Verwertungsinteresse einzelner Kapitale würde dazu führen, daß eine Reihe von Problemen, deren Lösung für die gesamtgesellschaftliche Reproduktion von entscheidender, für das einzelne Verwertungsinteresse aber von geringer Bedeutung oder sogar hinderlich wäre, der Wissenschaft zu spät oder überhaupt nicht gestellt würde.

$\mathrm{Da}$ dem Staat im Kapitalismus die Aufgabe zufällt, die notwendigen Reproduktionsbedingungen bereitzustellen, die das Kapital selbst nicht erstellt, weil dabei nicht genügend Profit gemacht werden kann, ist der Staat in bezug auf die Wissenschaft in dem Widerspruch verfangen: einerseits müssen die Interessen der Einzelkapitale an dem monopolisierten, gekauften Wissen gewahrt werden, weil es sonst über mangelnde Neuinvestitionen zur Wirtschaftskrise und letztlich zur Staatskrise käme, und andererseits der Notwendigkeit, solches privatisiertes Wissen der gesamtgesellschaftlichen Reproduktionssicherung zugute kommen zu lassen, die schließlich auch Existenzbedingung des Gesamtkapitals ist. Diesem Widerspruch gab der bürgerliche Staat durch die Einrichtung der öffentlichen, staatseigenen Universität auf der einen, und die Einrichtung des Urheber- und Patentrechts auf 
der anderen Seite eine Bewegungsform 10).

c) Bei so extremer Arbeitsteilung zwischen Ausformulierung der Theorie und ihrer praktischen Anwendung ergibt sich ganz allgemein folgende Schwierigkeit. Damit die Problemlösung nicht nur von privater Relevanz, sondem von gesellschaftlicher Bedeutung ist, muß sie die Antwort auf eine gesellschaftlich gestellte Frage sein. Dazu ist Voraussetzung, daß der Wissenschaftler in Kontakt ist mit den Bereichen, wo sich relevante d. h. für die Produktion und Reproduktion des gesellschaftlichen Lebenszusammenhangs notwendige Fragen ergeben. Je isolierter der Wissenschaftsbetrieb von den Bereichen der materiellen Produktion und Reproduktion und ihrer institutionellen Sicherung, desto größer ist die Wahrschein. lichkeit gesamtgesellschaftlich irrelevanter Fragestellungen.

Unter kapitalistischen Bedingungen stellt sich diese Schwierigkeit fiir das Kapital dann noch mit besonderer Schärfe, wenn die universitäre Wissenschaft nicht direkt dem Kapital untergeordnet ist, sondern nur indirekt beeinflußt werden kann. Da aber - wie sich schon unter Punkt b gezeigt hat - eine gewisse Autonomie der Universität wenigstens in Forschungsfragen aus wissenschaftsimmanenten Gründen notwendig ist (denn die Qualifikation von Forschern oder die wissenschaftliche Relevanz eines Forschungsprojektes kann von Nicht-Fachleuten nur ungenügend beurteilt werden, ohne Gefahr zu laufen, durch zu strenge äußere Eingriffe das Innovationspotential der Universität unnötig einzuschränken), beschränkt sich selbst der Staat im allgemeinen darauf, einen gewissen Zusammenhang zu den Problemen, die sich ihm als relevant darstellen und die daher eben die Probleme der bürgerlichen Gesellschaft sind, dadurch herzustellen, daß er sich die Entscheidung in der Personalpolitik und der finanziellen Forschungsförderung vorbehält.

Die daraus resultierende relative Autonomie der Universität machte es möglich, daß in der Folge der Studentenrevolte auch Wissenschaftler an die Universität kamen, denen sich die zu bearbeitenden Probleme nicht nur aus dem Zusammenhang der Erhaltung der bürgerlichen Gesellschaft, sondem aus dem objektiven Interesse des Proletariats an der Aufhebung dieser bürgerlichen Gesellschaft stell te. Diese marxistischen Wissenschftler mußten feststellen, daß die Universität schon so lange für bürgerliche Zwecke monopolisiertowar, daß selbst die Arbeitsmittel (Bibliotheken, Archive) auf deren einseitig, beschränktes Relevanz. kriterium hin ausgewählt und für Problemzusammenhänge im objektiven Interesse des Proletariats nur beschränkt verwendbar sind.

Die Orientienung vieler Studenten und einiger Dozenten auf dicse für

10) Das industrielle Mäzenentum in Großbritannien, USA u. Frankreich, das dort einen großen Teil der Universitäten trägt, ist nur eine andere Art u. Weise, diesen Widerspruch in einer tragbaren Form aufzufangen: Ist eine Erfindung privat nutzbar, so tritt das Mäzenenverhältnis außer Kraft u. wird durch ein Arbeitgeberverhältnis kurzzeitig ersetzí, $\mathrm{b}$ is sich gesamtgesellschaftliche Notwendigkeiten krisenartig geltend machen. Vgl. Nitsch, W.; Hochschule - Soziologische Materialien; Heidelberg 1967, S. 13 f.

Die Notwendigkeit einer Übernahme der Produktion von neuem Wissen durch größsere Einheiten als Einzelkapitale, vorzugsweise durch den Staat, ergibt sich auch daraus, daß sie für Kapitalisten ein höchst unsicheres, weil kaum prognostizierbares Geschäft ist $u$. daher auf industrieller Basis nur sehr schwer betrieben werden kann. 
bürgerliche Begriffe irrelevante und flugs für ,unwissenschaftlich erklärte Problemzusammenhänge is t neben dem Zwang zur Ökonomisierung von Ausbildung und Forschung ein Grund für die Einschränkung der Autonomie der Universität in allen neuen Hochschulgesetzentwürfen: über Fachaufsicht und Staatseingriff soll sich das Relevanzkriterium der bürgerlichen Gesellschaft wieder voll durchsetzen.

1.4. Die Frage, ob universitäre Wissenschaftler Kleinbürger sind und ihr Bewußtsein daher einfach als das von Kleinbürgern zu behandeln ist, beantwortet sich für die Universität als Institution, an der Forschung betrieben wird, so: zwar kann das neu produzierte Wissen zur Ware und daher Wissenschaftler zu einfachen, selbständigen Warenproduzenten, also Kleinbürgern, werden; im allgemeinen aber - und erst recht als universitäre Wissenschaftler - sind sie staatliche Angestellte oder Beamte, also Menschen, die zu ihrer Reproduktion auf den Verkauf ihrer Arbeitskraft angewiesen sind 11).

Trotzdem können Einzelne zeitweilig zu einfachen Warenproduzenten werden, wenn ihr neu produziertes Wissen patent- oder urheberrechtlich ausgewertet werden kann. Dies gilt praktisch aber nur für die technische Intelligenz. In den nichttechnischen Disziplinen bezieht sich das Urheberrecht wirtschaftlich nur auf die Druckrechte, nicht aber auf die Wiederverwendung der Problemlösung - also auf das Wissen selbst.

Trotzdem - und hier liegt das Richtige in der Analyse von Steffen und Funken - verhalten sich die Wissenschaftler in allen Forschungsbereichen in der wissenschaftlichen Diskussion zu ihren eigenen Ergebnissen und denen anderer wie zu Waren, die zum ,Gedankenaustausch" in die Zirkulation gegeben werden und deren Menge und, Wert ${ }^{6}$ - wie im zweiten Teil noch genauer zu zeigen sein wird Ger Ansehen und Einkommen des Produzenten entscheiden.

Dies äußert sich am deutlichsten in einem ritualisierten Urheberrechtsschutz auch gegenüber dem Wissen selbst, nämlich der Brauch, bei Verwendung einer Problemlösung den Namen des Urhebers mit zu nennen. Dieser Brauch macht das Wissen selbstverständlich nicht zur Ware, denn es findet kein echter Tauschakt statt,

11) Demnach wären sie Teil des Proletariats (vgl. erste Anmerkg. von Engels zur englischen Ausgabe der „Manifest der Kommunistischen Partei“ 1888, Marx/Engels Werke, Bd. 4, S. 462). Aber die Frage, ob ein Wissenschaftler einfacher Warenproduzent, Lohnarbeiter oder staatlicher Angestellter ist, hat auf die Bestimmung der Rahmenbedingungen seines möglichen Bewußtseins - übrigens ähnlich wie die vieldiskutierte Frage, ob er produktiver oder unproduktiver Arbeiter ist - nur insofern Bedeutung, als in ihrer Beantwortung ein Maß fur die relative Ferne zum Kapital, für den Grad der Subsumption unter das Kapital gegeben ist, aus dem zurückgeschlossen werden kann auf die Wahrscheinlichkeit sinnlicher Erfahrungen mit der Kapitalherrschaft. Das muß aber keineswegs bedeuten, daß3 die direkt subsumierten Wissenschaftler deswegen bessere Einsicht in den Charakter der Kapitalherrschaft oder sin antikapitalistisches Bewuistsein entwickeln müssen. Im Gegenteil: Da die direkte Unterordnung unter das Kapital - et wa in der chemischen Industrie - vor allem und zuerst einmal verschärtten Zwang zur Produktion von Problemlösungen u. damit verschärfte Konkurrenz zwischen den Wissenschaftlern bedeutet, wird dic kritische Kommunikation ijber die eigene gemeinsame Lage unwahrscheinlicher, die isoliert angestrengte Fachidiotie mit Imponiergehabe dagegen wahrscheinlicher. 
doch ist dieser Brauch Ausdruck einer strukturellen Analogie in der Verbreitung des Wissens zur Warenzirkulation. Die Analogie selbst hat ihre reale Basis darin, daß mit der Entwicklung der bürgerlichen Gesellschaft, d.h. mit der Entwicklung der Warenproduktion, alle Formen des Verkehrs zwischen den immer mehr zersplitterten Einheiten durch die verdinglichte Herrschaft des Produktes über den Produzenten und die Illusion der Autonomie des so beherrschten ,Individuums' geprägt wird. So scheint es, daß die wissenschaftliche Erkenntnis allein dem genialen Individuum Goethe, Einstein, Hegel zu verdanken sei, und mit der Angabe des ,Urhebers ${ }^{6}$ werden zwei Zwecke erfüllt: Erstens wird dadurch kenntlich, was ,fremde‘, was ,eigene Erkenntnis ist; zweitens soll so der relevante Produktionsausstoß eines jeden Wissenschaftlers festgehalten und damit ein Maß für seinen Platz in einer Prestigehierarchie festgesetzt werden, die von nicht geringem Einfluß auf sein Einkommen sein kann 12).

\subsection{Der Ausbildungsprozeß}

2.1. Das zweite Produkt, das an der Universität produziert wird, ist eine erhöhte Qualifikation der Studenten.

2.1.1. Die Studenten, die da qualifiziert werden, sind später in ihrer Berufsposition auf alle Klassen verstreut: Ein Teil wird Kapitalfunktionen ausüben, ein anderer Teil wird als Richter, Offizier oder Geheimdienstler direkt an der repressiven Herrschaftsstabilisierung durch den Staat teilhaben, wieder andere werden als Rechtsanwälte, frei praktizierende Ärzte und Steuerberater genuin ökonomische Kleinbürger sein. Zur Vereinfachung der Analyse wollen wir im folgenden von den Verhältnissen ausgehen,die für die große Masse der Studenten gelten, nämlich, daß sie ihre Arbeitskraft später als Ware verkaufen müssen 13).

2.1.2. Der Ausbildungsprozeß bildet konkrete Fähigkeiten in dem Arbeitsvermögen aus und macht die Arbeitskraft damit fähig, in komplizierten und komplexen Produktionsprozessen, Verwaltungs- und Aufsichtstätigkeiten eingesetzt zu werden. Die Qualifikation betrifft also zuerst die konkrete Seite, die Gebrauchwertseite des Arbeitsvermögens.

2.1.3. Diese Veränderung des Gebrauchswertes der Arbeitskraft verursacht aber Kosten, und zwar sowohl auf Seiten der Ausbilder und der Ausbildungsinstitution wie auf Seiten der Ausgebildeten. Da sich der Wert der Ware Arbeitskraft bekanntlich nach der zu ihrer Produktion und Reproduktion notwendigen Arbeit, also den dazu verauslagten Kosten bestimmt, ist der Ausbildungsprozeß zugleich mit einer Steigerung im Wert der Ware Arbeitskraft verbunden, was zugleich - und

12) Dies gilt besonders bei Lehrstuhlinhabern und solchen, die es werden wollen, wenn sie in Berufungs - und Bleibeverhandlungen eintreten. Aber auch sonst gibt es vielerlei Möglichkeiten: Referententätigkeit bei Stiftungen, Tagungen; Mitgliedschaft in Kuratorien, Beiräten und Expertenkommissionen, Autorenschaft, Zweitdrucke in Readern; Teilnahme an internationalen Kongressen; Fernsehauftritte, Zeitungsartikel etc., Beraterverträge usw., usw. - viele Möglichkeiten, die sich um so leichter erschliełben, je häufiger man gedruckt und zitiert wird.

13) Vgl. „Zur Klassenanalyse d. Studenten“ Bericht des Klassenanalyseprojektes, Oktober 1972, broschürt, 2. Auflage, Erlangen 1973. 
dies ist im Kapitalismus relevanter - einen Abzug am Mehrwert bedeutet.

2.2. Auch die Tatsache, daß die Ausbildung mit einer Wertsteigerung der Ware Arbeitskraft verbunden ist, macht die Dozenten noch nicht zu Warenproduzenten oder gar zu einfachen Warenproduzenten, denn die Qualifikation der Arbeitskraft ist nicht bloße Weiterverarbeitung irgendeiner Ware, sondern mit einer Reihe von wichtigen Unterschieden behaftet. Wären die Dozenten einfache Warenproduzenten, dann müßten sie gegen den Verkauf ihrer Ware ein Äquivalent eintauschen. Die Ware, deren Wert im Ausbildungsprozeß an der Universität durch die Dozenten erhöht wird, verkaufen aber nicht die Dozenten, sondern die ausgebildeten Lohnarbeiter selbst.

Wie also kommen die Ausbilder zu ihrem Wertäquivalent? Die Verhältnisse lassen sich am einfachsten zeigen, wenn man ein privat-kapitalistisches Ausbildungssystem annimmt (wie es heute noch in den USA in den meisten Colleges existiert): die Studenten zahlen hohe Schulgelder und Zulassungsgebühren, die den Preis darstellen für die Dienstleistung, die sie dafür erhalten. Die Verhältnisse sind hier noch nicht anders als z. B. beim Frisör: Die Dienstleistung befriedigt ein Bedürfnis, ist also Gebrauchswert und hat - da sie gegen Geld getauscht wird - Warencharakter. Die Studenten kaufen diese Dienstleistung jedoch nicht nur ein, um das Bedürfnis nach höherer Qualifikation, also verbesserter Problemlösungsfähigkeit zu genießen, sondern sie wollen dadurch beim Verkauf ihrer Arbeitskraft einen höheren Preis erzielen. Aufgrund der Illusion, er werde für seine Leistung und nicht für seine Reproduktionskosten bezahlt, also wegen der Fetischisierung des Lohnes aus dem Preis der Arbeitskraft in den Preis der Arbeit selbst, kann der Student gar nicht merken, daß er dem späteren Käufer seiner Arbeitskraft lediglich die höheren Kosten seiner Ausbildung in Rechnung stellt 14). Aber auch hier treten die Interessen eines Einzelkapitalisten, der sein Kapital in der Ausbildungssphäre angelegt hat, in Widerspruch mit dem notwendigen, durch den Staat getragenen Interesse des Gesamtkapitals an billigen Arbeitskräften, die zugleich die für die kontinuierliche Aufrechterhaltung des gesellschaftlichen Produktions- und Reproduktionsprozesses notwendigen minimalen Fähigkeiten besitzen. Der Staat muß daher Mindeststandards für die Ausbildungsstätten vorschreiben, und hat in der Folge die Ausbildung in nahezu allen Bereichen ganz übernommen.

Während bei der Privatuniversität die Bezahlung für die Dienstleistung „Ausbildung“ direkt aus den Zulassungsgeldern der Studenten kommen, die dann ihrerseits diese Summen den späteren Käufern ihrer Arbeitskraft in Rechnung stellen, muß der Wertausgleich in einer staatlichen, gebührenfreien Universität über die aus den höheren Einkommen gezahlten höheren Steuern laufen, die aber zu einem großen Teil über Umwege an die nicht-akademisch gebildeten Proletarier ,überwälzt ${ }^{\natural}$ werden.

2.3. In dem Bereich, wo die Dozenten den Wert einer Ware, die zentrale Stellung in

14) Diese Betrachtung wird belegt durch die vielfältigen Berechnungen, dats es sich im Durchschnitt nieht lohne zu studieren, wenn man die aufgewandten Kosten zusammen mit dem versäumten Lohneirkommen auf das Lebenseinkommen des Akademikers anrechne und es dann mit dem cines qualifizierten Industriearbeiters vergleiche. 
der Zirkulation hat, beeinflussen, in der Ausbildung, liegt in Deutschland bisher aber groteskerweise für sie selbst und ihre individuelle materielle Reproduktion als Universitätsdozenten die geringste Relevanz: Weder werden sie für ihre Funktion als Ausbilder - über das Vorexerzieren eines wissenschaflichen habitus hinaus - selbst ausgebildet, noch werden sie je auf den Erfolg ihrer Ausbildungstätigkeit uberprift, es sei denn abstrakt durch das Ausbleiben von ,Hörern' (und fruher Kolleggeldern), die man sich als Lehrstuhlinhaber aber wieder per Prüfungsordnung selbst verschreiben kann. Im Gegenteil, wenn sie als Ausbilder versagen, wird das demi Studenten oft genug von den unfähigen Dozenten selbst - in der Prüfung als deren Versagen angelastèt. Die Institution Universität sieht lediglich darauf, daß das formale Lehr. angebot erfullt ist. (Diese Ineffizienz und die damit verbundenen hohen Kosten der Ausbildung sind Gründe dafür, daß das Kapital in einige $n$ Bereichen, in denen sowieso schon große Tèile der Anlagen und Stipendien durch die Industrie finanziert werden, die Ausbildung auch auf Universitätsebene selbst organisieren will, und zwar weniger als profitable Warenproduktion denn als ein Mittel der Kostensenkung und der ,Optimierung ${ }^{6}$ der Personalpolitik).

2.4. Die Rolle der Studenten im gesellschaftlichen Reproduktionsprozeß ist die von im Qualifikationsprozeß stehenden zukünftigen Arbeitskräften. Dabei wäre es irreführend, die Studenten als einfache Warenproduzenten anzusprechen, da sonst schon das lernende und spielende Kleinkind so definiert werden müßte und auch z. B. ein Industriearbeiter bei der Ausbildung seiner Arbeitskraft bis zu ihrem erstmaligen Verkauf zu den Kleinbürgern, nach dem Verkauf zum Proletariat und während einer eventuellen Umschulung wieder zu den Kleinbürgern gerechnet werden müßte. Darüberhinaus befriedigt der Ausbildungsprozeß nicht nur die Bedürfnisse des späteren Käufers der Ware Arbeitskraft, sondern zuerst einmal die Bedürfnisse des Arbeitenden selbst.

3.0. Es gilt also festzuhalten: Es ist völlig unangebracht, die universitäre Intelligenz (Studenten und Dozenten) der ökonomischen Funktion nach dem Kleinbürgertum zuzuordnen. Wenn diese ursprünglich klassenanalytische Kategorie nicht zum bloßen politischen Schimpfwort wie Philister, Revisionist, Spießbürger, Seminarmarxist etc. verkommen soll, kann sie im Zusammenhang mit universitären Intellektuellen nur verwandt werden, um auf die strukturelle Analogie im Verhalten zum Produkt der Arbeit bei genuin ökonomischen Kleinbürgern und universitärer Intelli. genz zu verweisen. Dabei darf aber nie vergessen werden, daß es eben blok eine Analogie ist und daß die Folgen für die Arbeits- und Bewußtseinsformen durchaus unterschiedlich sind.

\section{Teil II: Die Arbeits- und Bewußtseinsformen der an der Universität ar- beitenden Gruppen}

\section{A. Zusammenfassung der bisherigen Ergebnisse}

Wir haben jetzt die allgemeine Analyse der Rolle der universitären Intelligenz im gesamtgesellschaftlichen Reproduktionsprozeß auf begrifflicher Ebene vorgenommen und dabei die Formen der universitären Produktionsprozesse nach den 
beiden Seiten des Arbeits- und Wertbildungsprozesses als Bedingungsgefïge für besondere Verhaltensdispositionen und Einstellungen grob eingegrenzt. Außer der scharfen Ablehnung, universitäre Intelligenz weiterhin pauschal als Kleinbuirger einzustufen, können wir zur näheren Bestimmung des spezifischen Rewußtseins universitärer Intelligenz von folgenden Ergebnissen ausgehen: 1. Das spezifische Produkt des universitären Produktionsprozesses ist - ganz allgemein gesprochen Wissen, und zwar in zwei Formen, einmal als neu produziertes Wissen: Neue Problemlösungen vornehmlich als Resultat der Forschungsprozesse; zum anderen vermitteltes oder angeeignetes Wissen: Inhalt und Ziel des universitären Ausbildungsprozesses als Qualifikation der Ware Arbeitskraft. Die zentrale Rolle spiels also das Wissen, wobei wir unter Wissen nicht nur abfragbare Tatsachen, sondern vor allem das Beherrschen von Problemlösungen und Problemlösungsverfahren meinen 15).

2. Das Wissen wird zwar nur unter ganz besonderen Bedingungen zur echten, $d, h$. gegen Geld direkt austauschbaren Ware, Bedingungen, die zudem an der Universität bloß als Ausnahme auftreten, doch besteht auch da eine strukturelle Analogie zwischen den Zirkulationsformen der Waren und den wissenschaftichen Formen, das Wissen zu behandeln und durch ,Gedankenaustausch zu kommunizieren. Da das Wissen für die Dozenten und Studenten darüberhinaus auch noch der Schlissel ist, der ihnen den Zugang zu den Mitteln der individuellen materiellen Repro. duktion verschafft, erscheint es als berechtigt, diese Seite des Wissens, im Kontrast zur unzweifelhaft vorhandenen Gebrauchswertseite, entsprechend, die Tauschwertseite des Wissens ${ }^{66} \mathrm{zu}$ nennen.

\section{B. Weiterführung der Fragestellung}

Nun können wir die Frage beantworten, die wir in den methodischen Überlegungen zur Ermittlung des Bewußtseins der universitären Intelligenz als die zentrale Frage gekennzeichnet haben: Welche Einstellungen und Verhaltensdispositionen erhöhen die Wahrscheinlichkeit von Erfolg im universitären Bereich? Also für die Studenten: Welches Verhalten führt zu guten Übungs- und Prüfungsergebnissen und gutem sozialem Ansehen bei Dozenten und Kommilitonen? Für die Dozenten: Wie kommt man bei denjenigen, die über Bewerbungen und Rufe entscheiden, in den Ruf ein erfolgreicher und solider Wissenschaftler zu sein?

15) Wir müssen uns hierbei Klar sein, dab dieser Wissensbegriff Resultat eines historischen Abstraktionsprozesses ist, der sich gegen das kanonische Wissen wendet, das bis vor kurzem in seiner Enzyklopädik den Bildungsbegriff bestimmte. Die Verselbständigung der Methode gegen den Inhalt - zum Ausbildungsprinzip erhoben in dem Slogan vom "Lernen lernen" - ist bedingt durch den rasanten Wandel der Berufsanforderungen im Kapitalismus, der ein einmal in der Jugend erlerntes Wissen innerhalb einer Generation mehrfach entwerten würde.

Dabei verändert sich aber auch der Begriff von dem, was Methode ist, der bei Hegel und Marx noch die Darstellung und Entfaltung des konkret-inhaltiichen Stoffes meint (,,Denn die Methode ist nichts anderes als der Bau des Ganzen in seiner reinen Wesenheit aufgestellt" Hegel-Vorrede zur Phänomenologie) zur rezeptartigen Handlungsanweisung, die von Hegel als „,dem Stoffe äußerlich" kritisiert wird. 
Allgemein: Mit der detaillierten Beantwortung der Frage nach den spezifischen Einstellungen und Verhaltensweisen, die im Bereich der Universität dazu führen, daß die individuelle Reproduktion für die Zeit des Aufenthaltes an der Universität auf materieller, sozialer und psychischer Ebene verbessert werden kann, mit dieser Antwort haben wir die Bewußtseinsformen und Verhaltensweisen herausgefunden, die spezifisch durch die Institution Universität gefördert werden und die man daher jenseits aller Unterschiede in der individuellen Sozialisation den „Subjektiven Faktor" der universitären Intelligenz nennen kann.

$\mathrm{Da}$ an der Universität im allgemeinen keine Waren produziert werden, durch deren Verkauf die Produzenten ihren Unterhalt verdienen, müssen irgendwelche andere Kriterien bestehen, die darüber entscheiden, wer unter welchen Bedingungen an dieser Institution tätig sein und damit aus dem über Steuergelder und Stipendienfonds bereitgestellten Teil des gesellschaftlichen Gesamtproduktes anteilig schöpfen darf. Diese Kriterien - so irrational und unausgewiesen sie immer sein mögen - entscheiden damit über den materiellen und sozialen Erfolg an der Universität, sind also erste Antwort auf unsere Frage.

Für die Studenten sind die formalen Kriterien in den Prüfungs- und Studienordnungen festgelegt. Dabei ist es immer weniger relevant, ob ein Student sein Geld von seinen Eltern oder aus einem Stipendienfonds bekommt - beide richten sich primär nach den Ergebnissen der Übungen und Prüfungen. Allerdings ist derjenige Student, der durch seine Eltern finanziert wird, noch einer Reihe zusätzlicher, oft höchst irrationaler Kriterien ausgesetzt, die aber nicht universitätsspezifisch sind.

Auch bei den Dozenten sind die formalen Kriterien, die über den Aufstieg entscheiden in Prüfungsordnungen festgelegt. Davon sind vor allem diejenigen Dozenten betroffen, die noch keinen Lehrstuhl auf Lebenszeit besitzen. Aber auch die Lehrstuhlinhaber sind über den Zwang, mit ihren Kollegen um Rufe zu konkurrieren, einem zwar weniger formalisierten aber keineswegs weniger nachhaltigen Druck ausgesetzt - auch wenn sie sich nicht um Weltruhm bemühen. Dieser Druck wird denn auch oft genug als ,Erziehung zur Wissenschaftlichkeit" nach unten an Assistenten und Studenten weitergegeben.

Auf formaler Ebene entscheiden also Prüfungen unterschiedlicher Art (Übungen, Examina, Promotion, Bewerbungen) über Aufstieg und Verbleib an der Universität. In diesen Prüfungen soll - laut Vorschrift - der erreichte Grad der Qualifikation festgestellt und verglichen werden: Das bedeutet vor allem eine Prüfung des Wissens, und zwar müssen gestellte Probleme in einer durch den Prüfenden anerkannten Weise gelöst werden; neben dem Umfang des reinen Faktenwissens wird also Problemlösungswissen zum Kriterium über Verbleib und Fortkommen an der Universität. Bei den Dozenten kommt noch das Kriterium hinzu, wie viel neues Wissen sie selbst produziert und publiziert haben.

In diesen Kriterien macht sich die oben entwickelte "Tauschwertseite" des Wissens geltend: Unterschiedliche Qualitäten müssen auf Quantitäten reduziert we fden, um zwischen unterschiedlichen Bewerbern auswählen oder sie in eine formal abgestufte Leistungshierarchie einpressen zu können. Im Unterschied zur Wertgröße gibt es hier aber kein allgemein gültiges, objektives $\mathrm{Maß}$, vielmehr wird dieses $\mathrm{Maß}$ 
subjektiv durch die Prüfenden gesetzt: Man muß auf alle relevanten - und wo: man wirklich gut sein will, auch auf alle irrelevanten Fragen (man weiß ja nie, was der Prüfer für relevant hält) - eine Antwort bereit haben, die - und das ist dss wichtigste Kriterium - den Fragenden befriedigt, und zwar oft genug in der voles. Breite des Wortes, da in dem Verhältnis zwischen Prüfer und Geprüftem eine Unmenge von unbewußten und unkontrollierten Bedürfnissen und Angsten mitspieit. So erweist sich, daß unter dem rationalen Schein der formalen Prüfungskriterien nicht nur die Möglichkeit zur dogmatischen Diskriminierung unbequemer wissen. schaftlicher Richtungen verborgen ist - etwa indem alle marxistisch beantworteten Fragen a priori als unwissenschaftliche und bloß dogmatische Ideologie definiert werden - sondern darüber hinaus ist dies formal rationale Kriterium die Basis für Irrationalität: Statt wissenschaftlicher Qualifikation wird tendenziell blober wissenschaftlicher habitus geprüft und gezüchtet - man muß dem Prüfenden auf wissenschaftliche Weise hochkriechen.

Diese oft als ,Ungerechtigkeit ${ }^{6}$ kritisierte Irrationalität hat aber durchaus thren Sinn: der Zwang zur Anpassung an die Erwartungen des Prüfers unterwirft nur dem äußeren Schein nach unter die Willkür eines irrationalen Einzelmenschen. In Wirklichkeit repräsentiert der Prüfer bürgerliche Durchschnittserwartung, denn er ist selbst nur dadurch an diesen Platz gekommen, weil er die Prüfungen bestanden, die Erwartungen erfült hat. Die scheinbar irrationalen Prüfungen stellen also die Warenrationalität der Aquivalenz des durchschnittlich Gleichen für den Bereich der Universität her, was seinen äußerlich sichtbaren Ausdruck findet, in den Verhandlungen der Kultusminister und Universitäten um gegenseitige Anerkennung der Prüfungen. Die Prüfungen erhalten so die Form von Etiketten auf die verkaufbare Ware - und, ganz entsprechend der strukturellen Analogie, wird nichts so gefürchtet wie der „Etikettenschwindel“ der darin besteht, daß Marxisten sich mit dem bürgerlichen Prüfungsetikett in die Stellenzirkulation, schleichen".

Der Zwang zur Anpassung herrscht aber nicht nur an der Universität, er ist also nur insofern universitätsspezifisch als er sich auf das Verhalten gegenüber dem spezifischen Produkt der Universität auswirkt: Das, was gewußt werden muß, bestimmt sich nicht mehr primär an dem Bedürfnis, inhaltliche Probleme zu lösen, sondern orientiert sich stärker an der äußerlichen sozialen Bezießung zwischen Fragendem und Befragtem. Das sozial erwartete Wissen wird zum relevanten Wissen; die soziale Erwartung ersetzt teilweise das oben entwickelte Relevanzkriterium des Bezuges zur materiellen Reproduktion, das seinen Platz objektiv nur darum halten kann, weil die soziale Erwartung eben nicht bloße individuelle Subjektivität, sondern eben auch durch die Vermittlungszusammenhänge zur materiellen Reproduktion bedingt ist.

$\mathrm{Da}$ in den Prufungen also letztlich der Umfang des sozial erwarteten Problemlösungswissens entscheidet und da das, was sozial an Wissen erwartet wird, nie voll bekannt ist, wird es zur Notwendigkeit, möglichst viel Wissen arzuhäufen, denn man kann sich nie sicher sein, daß man genügend weiß und ob das, was man weiß, auch gerade den Erwartungen entspricht. Diese Notwendigkeit wird dadurch noch drängender und beständiger, daß der Umfang des Wissens nicht nur crch die Erwartungen in den Prüfungen, sondern auch durch die Erwartungen in der sonsti- 
gen sozialen Kommunikation im universitären Bereich bestimmt ist. Das heißt, das Sozialprestige - und bei denjenigen, die keine sonstigen Bewähnungsfelder haben, auch rock das Selbstwertgefihl - hängt ab vom Umfang und der Weise der Frăsentation des jeweils sozial als relevant erachteten Wissens.

Man könnte demnach die universitäre Intelligenz genauso, wie sie mit Klembiurgern in Analogie gesetzt wird, auch mit Schatzbildnern oder noch besser mî Kleinbauern vergleichen: Je größer der Misthaufen vor dem Iaus, desto größer das Ansehen im Dorf.

Wirkliche Schatzbildung ist die Ansammlung von Geld, mit denn nicht eingekove wird, sie verhindert den Konsum - im Sprichwort: Man kann den Kuchen ncht haben und zugleich essen wollen. Mit dem aufgehäuften Wissen verhält es sich aber ganz anders: Man kann nicht nur uber Wissen verfugen und dieses Wissen durchaus zugleich verwenden, also den Kuchen haben und ihn zugleich essen, sontem man kann das Wissen auch bloß vortäuschen und - jedenfalls was die Tauschwertseite des Wissens angeht-den gleichen Effekt erzieler, als ob man wirklich wiste: Man kann den Kuchen also essen, ohne ihn überhaupt zu haben.

Das Vortäuschen des Wissens ist möglich, weil in Prüungen und Gesprächen nicht die konkret-qualitative, tatsächliche Problenlösung entschsidend ist, sondern beim Gesprächspartner, der Prüfer ist, lediglich der Eindnck entstehen muB, das geforderte Wissen sei vorhanden. $\mathrm{Da}$ es also nur darauf ankormt, den Fragesteller zu befriedigen, genügt es, den Anschein von Wissen zu erzeugen: So wie man kreditwïrig sein kann, wenn man den Anschein von Reichtum erwecken kann, so ist es auch möglich, an der Universität die Kriterien über Verbleib und Fortkommen zu einem guten Teil durch Bluff zu erfillen. In den sozialen Beziehungen ist das dann noch viel einfacher.

Die Möglichkeit des Bluffs wird sofort zur beinahe allgemeinen Notwendig. keit, denn die qualitative Unendlichkeit dessen, was möglicherweise gefordert werden könnte, wird nun nicht mehr ausgewogen durch das Wissen um die Grenzen dessen, was man sich z. B. als Erstsemester bisher hat erarbeiten können. Die Möglichkeit, schon im ersten Semester mit einem erfundenen oder aufgeschnappten Zitat aus den 2. Band von Hegels Ästhetik den Eindruck eines Hegelkenners machen $z u$ können, sprengt alle solchen Grenzen und - da der Bluff auf alle, außer den Bluffenden echt wirkt-wird die quantitative Anforderung auf allen Stufen unmäßig verschärft - so sehr, daß sie schließlich niemand mehr anders als durch Bluff erfuillen kann. Unter diesen Umständen können nur extrem Ichstarke noch auf den Buff verzichten - oder solche, die in wenigstens einem Gebiet die Anforderungen tatsächlich erfullen und immer wieder darauf verweisen können 16).

16) Die vielfältigen Formen des Bluffs sollen hier nicht beschrieben werden (das kann dem Selbststudium des Lesers überlassen bleiben - womit schon eine Form vorgefuhrt wäre!), aber es muß doch differenziert werden zwischen mündlicher und schriftlicher Form des Bluffens: Da das Wesentliche am Bluff darin besteht, so zu formulieren, dah jede Nachprifung ausgeschlossen ist aber doch der Eindruck großen Wissens entsteht, ist der Bluff in schriftlichen Arbeiten sehr viel schwieriger anzubringen als im mündlichen Verkehr, denn die Wissenschaft hat alles, was nicht nachprüfoar ist, als unwissenschaftlich ausgegrenzt. Außer dem Plagiat, das sehr gewagt ist, und daher relativ selten 
Beinahe alle missen also biluffen. Aber eben nur beinahe alle. Daher weif keiner, ob der andere nicht vielleicht tatsächlich Bescheid weiß und den Bluff durchschauen, die Kreditwürigkeit zerstören kann. Das macht aber den Bluff noch schwieriger und erzeugt neben der allgemeinen Konkurrenzsituation, die sich allein schon aus der Hierarchie des Wissens ergibt, die ständige Angst vor dem anderen, der den Bluff durchschawen könnte. Diese Angst verschwindet erst, wenn klar ist, dak der andere zu wenig weil, um gefährlich zu werden. Da der andere aber eben dies furchten muf, muB er dic Kommunikation in Bahnen halten, in denen es auf keinen Fall zu einer solchen Entlarvung kommen kann. Ein Gespräch kann sich also nur als beinahe ritualisiertes, ganz vorsichtiges Abtasten entwickeln. Das Resultat ist gegenseitige Isolierung, die äußerlich meist als Arroganz erscheint 17).

Zu der allgemeinen Konkurrenzsituation kommt also noch eine Distanzienng, die jede Solidarität und erst recht jede libidinöse Beziehung erschwert. In der daraus folgenden Isolierung verschärfen sich unter dem verinnerlichten Druck der unerfullbaren Wissensanforderungen die individuellen Komplexe und Sozialisationsschwierigkeiten ins Katastiophale 18). Früher wurde versucht, diese Isolation durch die zeremonell ritualisierte Kommunikation in den akademischen Verbindungen aufzufangen. Eine andere Möglichkeit waren die Studentengemeinden. Beide Organisationstypen hatten daher großen Zulauf und haben es an vielen Universitäten auch nock hevte. Die anderen organisierten Hochschulgruppen politischer oder existentautistischer Richung blieben dagegen bis in die Mitte der sechziger Jahre im Vergleich schr klcin, dem sie waren vomehmlich auf intellektuelle Diskussionen ausgerichtec, die damit die Zwänge der universitären Konkurrenz wieder auf-

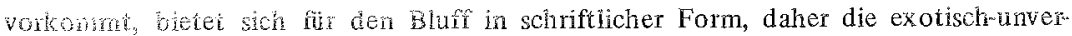
stänhlohe romulenug und - als wichtigstes und häufigstes Mittel - der wissenschafthohe Appatal an, dessen urspungliche Bestimmung es war, gerade die Nachprübarkeit zu geratinen. Migendwo wird so viel geblufft wie in Fußnoten und in Literaturerzelchuren.

17) Zwo mo 3 wag, Konkurenz ohne Freiheit zum Ausweichen und Spezialistentum

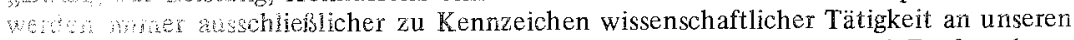
Hodriter -... Whet Womente, deren Reflex vielfach in Isolierung und Entfremdung, atrit, tas einem modemen Großbetrieb zu erkennen ist. Der Mensch leidet

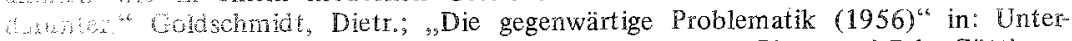
monumgen zur Lage der deutschen Hochschullehrer; Hrsg. H. Plessner, 3 Bde, Gö́tingen 1956; 1. Band \$. 40. ,Alles, was wir - direkt oder indirekt - tun, um unserer soriagngst zu entgehen oder sie zu verstecken, gehört zur Angstabwehrfassade. Indem Wir aber immer wieder diese Angstabwehrfassade aufsetzen (was kein bewußster Vorgang ist, sondern meist ganz unbewußst und automatisch geschieht), konservieren wir unsere Angst statí sie loszuwerden... Die Angstabwehrfassade erhält die Angst weil der Setreffende nie die Eriahrung machen kann, daß er auch mit seinen Fehlern und ohne sein Imponiergehabe akzeptiert wird von den anderen." Mannheimer Paper, a. a. O. S. 11 f.

18) „Von hunderttausend Studenten begehen jährlich durchschnittlich 25 Studenten Selbstmord, aber vergleichsweise nur 19 Personen der Gesamtbevölkerung und sogar nur 14 Personen der Altersgruppe 18-30 Jahre." (W. R. Morgenstern; ,Botschaft - Appeil - Flucht, Selbstmord und Seibstmordversuch bei Studenten" in: Analysen - Zeitschrift für Wissenschafts und Berufspraxis, 2. Jg. 1972, Nr. 1 Januar, S. 28. 
nahmen. Linke organisierte Gruppen, deren Mitglieder durch ihre individuelle Sozialisation, Bekanntschaften, moralische Empörung oder gar in Ausnahmefällen durch bloß intellektuelle Erkenntnis links geworden waren, gab es also schon lange vor der Studentenbewegung.

\section{Exkurs: Versuch, einen Aspekt der Studentenbewegung zu erklären}

Um den ,subjektiven Faktor"6 in der Studentenbewegung zu analysieren, ist es also kein Problem, die Existenz einer fuihrenden, aktiven Gruppe zu erklären, die aktuelle Vorgänge auf hohem Niveau analysierte. Das Problem ist vielmehr die sprunghafte Verbreiterung ihrer Basis in eine an ihrer Basis solidarische Massenbewegung - eine Massenbewegung unter Studenten, die nicht nur als absteigende Schicht für die Erhaltung ihrer Privilegien kämpfte, sondern zugleich mit dem Ziel auftrat, eine allgemeine Demokratisierung der Gesellschaft herbeizuführen, und bei der wesentlicher Inhalt ihres Entwicklungsprozesses der Kampf gegen den Imperialismus war. Die bildungsökonomischen Bedingungen dieses Prozesses sind bekannt: 19) Der äußere Druck durch $Z$ wischenprüfungen, numerus clausus und Studienzeitverkürzung erhöhte die Überforderung durch die Tauschwertseite des Wissens und zugleich würde es durch die Überfüllung der Veranstaltungen und den Mangel an Dozenten und Lehrmaterial immer schwieriger, sich die Gebrauchswertseite des Wissens zu erarbeiten.

Als die vorhandenen linken organisierten Gruppen in dieser Situation in Westberlin und anderswo die Auflehnung gegen die traditionell anerkannten Autoritäten zuerst in Veranstaltungskritiken, die den beschränkten Gebrauchswert dieser Renomierveranstaltungen aufzeigten, und schließlich gegen die ganze Institution, gegen Inhalt und Organisationsformen der Universität vortrugen, mußte sie schon allein dadurch zu einem Kristallisationspunkt werden. Als sie darüberhinaus durch politische Demonstrationen gegen den Imperialismus in Vietnam, im Kongo und in Persien und der Reaktion der Staatsorgane auf diese Demonstrationen das brutale Aus. einanderklaffen von Anspruch und Wirklichkeit der bürgerlichen Geselischaft verdeutlichte, konnte sie neben den in ihren Privilegien Bedrohten auch roch die moralisch Empörten und die intellektuell Beeindruckten 20) um sich sammeln.

Zur Massenbewegung spontaner und in sich für studentische Mafsstäbe recht solidarischer Aktionsgruppen, die die Basis der Studentenbewegung ausmact terk

19) Hier einige Literaturangaben zu Ursachen und Verlauf der Studentenbewegung, von denen ich auch wiederum die meisten nur weiterempfehlen kann: Zur Klassenanalyse der Studenten, Berlin (W), 1973; Marxistisch-Leninistische Hochschulgruppe Bau, Postionspapier, Berlin (W), 1971; Zur Entstehung und Geschichte der Studenten- und Schülerbewegung, Arbeiterpolitik, Bremen, 12/70; Thesen zur Studentenbewegung, Marxistisch-Leninistische Hochschulgruppe Germanistik, Berlin (W), 4/71; Internationale Studentenrevolte, Kursbuch 13, Frankfurt/Main, 1968

20) Schon 1968 führte die Beachtung durch einige anerkannte intellektuelle Autoritäten und einige ebenso anerkannte Zeitschriften dazu, daß die damalige Theorie der Neuen Linken ein wenig zum notwendigen, weil sozial akzeptierten Wissen wurde. (Ein Indiz: Der "Spiegel" fing an, in sinnigem und unsinnigem Zusammenhang Herbert Marcuse zu erwähnen). 
und sich als solche ,Basis"-Gruppen stakl won der fuhrenden Gruppen im SDS und SHB unterschieden, denn diese waren veitekin furchteregend intellektuell und daher hierarchisch strukturiert, zu disser Massenbewegung entwickelte sich die Studentenbewegung erst dadurch, dab sis anti-autoriâr auftrat: Damit waren die individuellen Mängel und Fehler - wie mangelndes Wissen, Orgasmusschwierig. keiten, die Aufgeregtheit beim Sprechen, das stane Liebesbedurfnis und das Leiden unter der Einsamkeit usw. nicht mehr bloß private Schvierigkeit, die man möglichst verbergen und überspielen mußte, wollte man Anerkenmung finden, sondern diese Schwierigkeiten wurden als gesellschaftich produzierte Feh haltungen erkannt, die auf der gleichen Ebene liegen, wie die faschistoide Disposition im autoritären Typ. Wenn man diese Schwierigkeiten vor der gleichgesinnten Gruppe erkemen ließ und darstellte, entlarvte man sich nicht mehr selbst oder erzeugte gar die peinliche Situation, in der alle sich zu der Bemerming gezwungen sehen: ; Ach so schlimm ist es doch gar nicht mir Dir". Anstatt sich selbst bloßzustellen, stellte man ,das System" bloß: Die eigene psychische Emanzipation war nicht nur Voraussetzung des Klassenkampfes, sondern erschien schon selbst als ein revolu tionärer Akt 21).

Unter diesen Vorzeichen war es möglich, in einem überfüllten universitären Seminar offen ohne Verlust an sozialem Ansehen die Hilflosigkeit gegenüber einem Problem zuzugeben. Damit war der Konkurrenzdruck zwar nicht abgeschafft, aber doch erheblich reduziert und damit auch der Bluff mit seinen isolierenden Folgen nicht mehr so notwendig. Damit schwand aber auch der Grund fur die Isolierung und Distanz zwischen den Studenten und mit dem Schwinden der Distanz konnten sie sich zu einer solidarischen Massenbewegung verbinden, in der sich die libidinösen Beziehungen einigermaßen angstfrei betätigen konnten, denn der ,Psychoterror, in den die solidarischen Diskussionen in den Wohngemeinschaften und Basisgruppen manchmal umschlugen, war weniger terroristisch, weil weniger isolierend, als das ritualisierte Spiel ,Wer-weiß-mehr-über-Hegel' der alten Universität. Die antiautoritäre Studentenbewegung entwickelte sich aber schon allein aufgrund der konkreten Seite der Auseinandersetzung mit der bürgerlichen Gesellschaft von den spontanen Selbstbefreiungszirkeln zu organisatorisch strukturierten Gruppen. Damit ging aber

21) Auch das Mannheimer Papier setzt hier die entscheidende Differenz zwischen Studentenbewegungen und klassenkämpferischer Politik an: Zitat S. 25 f. ,Auf eine einfache Formel gebracht, könnte man den Unterschied so ausdrücken: Der Student sieht den Klassenfeind größstenteils innerhalb, der Arbeiter außerhalb seiner eigenen Person. Wenn wir nun vernünftige Prinzipien der politischen Strategie ausarbeiten wollen, so dürfen wir dieses psychologische Unterscheidungsmerkmal unserer verschiedenen Klassenlage nicht übergehen. Wir sollten sehen, dafs dje Genossen aus dem Mittelstand erst dann zu radikalen Klassenkämpfern werden können, wenn sie den autoritären Feind (über-ich) in sich selbst überwunden haben. Um diesen inneten Feind aber zu überwinden, ist eine antiautoritär-emanzipatorische Phase notwendig .... Wir befürworten entschieden den Kampf gegen das eigene über-ich als eine notwendige Vorstufe des Klassenkampfes. Wir wenden uns aber dagegen, diesen Kampf bereits als Klassenkampf auszugeben. Wir müssen sehen, daf3 es sich hier um eine mittelständische Befreiungsbewegung handelt, die zwar bereits klassenkämpferische Elemente in sich trägt, die aber erst dann zum eigentlichen Klassenkampf wird, wenn sie sich - nach der emanzipatorischen Übergangsphase - umsetzt in revolutionäre Arbeiterpolitik." (S. 25 f). 
auch notwendig die Erarbeitung einer entwickelteren und wissenschaftlich geschärften Theorie einher. Da aber die bloße Auflehnung gegen die Ordinarienherrschaft den institutionellen Charakter der Universität als Stätte, die Wissen schafft und prüft, nicht verändem konnte, blieben die $\mathbb{Z}$ wänge der Universität voll wirksam und in dem Maßse, in dem der Kampf der Universität zur Nebensache und der Appell an die Libido als Riickfall in die antiautoritäre Phase erklärt wurde, mußten diese $Z$ wänge auch wieder auf die studentischen Angehörigen und Sympathisanten der linken Gruppen durchschlagen.

Allerdings war inzwischen der theoretische Wissensanspruch gegen die Linken noch viel totaler geworden als je zuvor: Die gesamte marxistische Theorie und mit inr die konkrete Totalicät der bürgerlichen Gesellschaft samt ihrer Interpretation durch die buirgerliche Wissenschaft soll kritisch begriffen sein. Dies ist aber nicht genug: Erst die revolutionäre Praxis gibt der Theorie ihren Stellenwert, und diese Praxis kann nur in enger, organisatorischer Verbindung mit dem Proletariat wirklich revolutionäre Praxis sein!

Vor diesen unerfillbaren theoretischen und praktischen Anspriichen war es unausweichlich, daß die alten, von der Universität als Institution weiterhin erzeugten Konkurrenz- und Isolierungsmechanismen sich auch innerhalb der Linken wieder durchsetzten. Damit fiel aber die spontane, weil libido-bestimmt agierende, solidarische Massenbasis in sich zusammen. Die Forderung nach Solidarität wird zur moralischen, abstrakten Forderung, die auch unter Genossen aufgesetzt wirkt - wje die Form der Auseinandersetzung immer wieder beweist. Vorwiegend dort, wo noch Gruppen außerhalb und gegen die Universität in Wohngemeinschaften und nicht-konkurrenzbezogenen Zusammenhängen bestehen, ist eine reale Basis für spontane Solidarität noch gegeben. Diese Entwicklung verschärft sich dort, wo die marxistische Theorie an der Universität selbst als Teil des normalen Lehrprograrmms gelehrt und gepruft wird. Dort besteht die reale Gefahr, daß das terroristische Spiel ,Wer-weiß-mehrüber-Hegel ${ }^{6}$ durch ,Wer-weiß-mehr-über Marx-Engels-Lenin-Mao etc. oder "Wer-ist-revolutionärer ${ }^{c}$ abgelöst wird. In den Lehrveranstaltungen reproduziert sich - dazu noch in formalisierter Weise - was in den politischen Gruppen längst doninant geworden ist: "Die stevernde Kraft für das, was gesagt bzw. verschwiegen wird, ist meistens nicht die politische Vernunft, sondern die Angst vor dem negativen Ureil der anderen, vor allem der Großgenossen ... Diese Angst ist oft verbunden mit einem Rivalentum, das mit seiner hintergründigen oder offenen Feindseligkeit genau den Leistungsterror und das Konkurrenzdenken unserer birgerlichen Gesellschaft reproduziert. Wir sind Konkurrenten im Kampf um die Aneignung von Prestige und Status innerhalb der Gruppe 22).“

Die Studentenrevolte konnte also darum zur im Ansatz solidarischen, spontanen Massenbewegung werden, weil sie zeitweise in kulturrevolutionärem Imperus die isolierenden und angst-verstärkenden universitären Konkurrenz- und Leisiungș wänge wenigstens zum Teil außer Kraft setzen konnte. In dieser Zeit entwickelte sich die Theorie der ,Neuen Linken" selbst zum sozialerwarteten Wissen, in einigen gesellschaftswissenschaftlichen Instituten sogar zu einem Teil des

Mannhemer Papier, a. a. O., S. 5. 
institutionell erwarteten Wissens. Jetzt, nachdem das kulturrevolutionäre Element völlig durch eine geradezu imitatorische Orthodoxie der Parteigründungen verdrängt worden ist, kann sich die Institution Universität wieder mit ihren besonderen bewußtseinsprägenden Zwängen voll auf Studenten, Dozenten und andere Dienstkräfte auswirken.

Bevor diese Auswirkungen im einzelnen dargestellt werden, müssen aber noch zwei wichtige Differenzierungen eingebracht werden: 1. Die unterschiedliche Wirkung in unterschiedlichen Fächern; 2. Die unterschiedliche Betroffenheit nach Geschlecht und sozialer Herkunft.

\section{Einige Differenzierungen}

\section{Differenzierung nach Studienrichtungen}

Die Auswirkungen der Institution Universität auf die Studenten werden ganz entscheidend modifiziert je nachdem welche Fachrichtung sie studieren: $\mathrm{Ob}$ es sich um den Grad und das Ausmaß der Beteiligung in der Studentenrevolte oder um die Häufigkeit psychischer Erkrankungen, oder um erfolglosen Abbruch des Studiums, die Häufigkeit von Fächerwechseln oder gar um die Häufigkeit von Selbstmorden und Selbstmordversuchen handelt, alle Untersuchungen zeigen immer wieder das gleiche Verteilungsmuster innerhalb der Fakultäten: Die Fächer der früheren Philosophischen Fakultät stehen überall mit der häufigsten Inzidenz deutlich an der Spitze, gefolgt von den Wirtschafts- und Sozialwissenschaften, dann den Naturwissenschaften vor der Rechtswissenschaft und der Medizin 23).

23) Vgl. Goldschmidt, D.: „Die objektive Studiensituation der Studierenden in der Bundesrepublik Deutschland als eine Stress-Situation" in: Psychische Störungen, a. a. O., S. 51-66; S. $56 \mathrm{ff}$; und Lungerhausen, E.: Selbstmorde und Selbstmordversuche bei Studenten; (Hüthig) Heidelberg 1968, S. 38 ff., und ders.: ,Zum Problem der Suicidhandlung an Universitäten“, in: Psychische Störungen, a. a. O., S. 103-111; S. 109: ,, Bei allen Untersuchungen zeigte sich immer wieder, daß - handelte es sich um psychische Belastungen, Krankheiten oder Mißerfolge im Studium - Das Verteilungsmuster innerhalb der Fakultäten weitgehend dem entsprach, das wir bei den Suiciden fanden." Dabei ergeben sich bei der statistischen Aufstellung von Lungerhshausen die Schwierig. keiten, dałs er die den Geisteswissenschaften verwandten Wirtschafts- und Sozialwissenschaften zusammen mit der Rechtswissenschaft zählt und dadurch zu einer anderen Reihenfolge kommt. Die Einteilung Goldschmidts wird jedoch gestützt durch die Beobachtungen von: Rohr, Ch.: „Psychologische Variablen bei der Untersuchung von Leistungstörungen bei Studenten" in: Psychische Störungen, a. a. O., S. 152-161, S. 154; Böker, W.: ${ }_{y}$ Psychische Probleme bei Studierenden. Symptomatik, Ursachen und Behandlungsmöglichkeiten ${ }^{\text {‘6 }}$ in: Zeitschrift für Psychotherapie und medizinische Psychologie, Stuttgart J. 19, 1969, H. 4, S. 137-153, S. 140. Bay, Ch.;,Political and Apolitical Students: Facts in Search of Theory ${ }^{64}$ in: Jorunal of Social Issues, Vol. 23, 1967, N. 3, p. 76-91, $82 \mathrm{f}$., sowie die meisten Fallstatistiken in "Psychische Störungen." Bei Lungershausen ist die Reihenfolge: „Innerhalb der Geisteswissenschaftlichen Fakultät am höchsten, ihnen folgen Naturwissenschaften, Medizin, Rechtswissenschaften, Wirtschafts- und Sozialwissenschaften und sehließlich die Studenten der technischen Fächer ..." Lungershausen: Selbstmorde...., S. 39. 
Der Grund für diese Úbereinstimmung in der Verteilung allgemeiner Krisenanfälligkeit und politischer Aktivität liegt wahrscheinlich einerseits darin, daß bereits besonders Strukturierte und Interessierte diese Fächer hoher Inzidenz wählen und andererseits, daß die Besonderheiten der Fächer solche Einstellungen und Verhaltensdispositionen in besonderer Weise fordem.

Für den ersten Faktor, die Selektion bei der Fächerwahl, stellt Lungershausen folgenden Erklärungsversuch auf:

„SStudenten der Medizin, der Rechts- und Wirtschaftswissenschaften, der Technik pflegen gewöhnlich ein festes Berufsziel zu haben. Sie benötigen die Universität nur, um die fur ihren $\mathbb{B}$ eruf notwendige Ausbildung zu erhalten und haben dabei den Vorteil, daf die Universität ihnen dazu ein weitgehend festgelegtes Programm bietet, das in bestimmten Etappen zuruckgelegt we rden muß. Diejenigen Studenten, die unentschlossen zögend, ihrer Lebenziele unsicher, das Studium beginnen, um dann später, weiter zu sehen ${ }^{6}$, werden von vornherein mehr zu anderen Fakultäten neigen ...., die, jedenfalls am Studienbeginn noch keine Festlegung hinsichtlich der weiteren Zukunft erfordern."

Diese präzisere Definiertheit des instrumentell für spätere Berufstätigkeit notwendigen Wissens ist damit zugleich ein Unterschied in der Spezifik der Fachrichtungen, der nach dem einmal begonnenen Studium die Verselbständigung des Wissens zum bloßen Mediun der Bildung von Sozialhierarchien beschränkt. In der Folge wird zwar in den stark berufsorientierten Fächern ein schulartiger Leistungsdruck die Studenten zum Stöhnen bringen, da aber das zu erarbeitende Wissen einigermaßen überschaubar und kalkulierbar ist, besteht kein so ausgeprägter Zwang zum Bluff und daher auch keine so ausgeprägte Konkurrenz und Isolierung unter den Studenten: Sie können gemeinsam stöhnen und sich um der kalkulierbaren Berufsperspektive willen anpassen. Meist ist damit aber nur eine besondere Form der Konkurrenz, nämlich der universitäre Bluff, reduziert. Dafür findet dann um so schärfer eine vorgezogene beruftiche Konkurrenz statt, die nur in der ritualisierten Kameradschaftszone der Verbindungen oder Verbindungs-ähnlicher Skatgemeinschaften zeitweise außer Kraft gesetzt werden kann.

Dazu kommt, daß ein dezidiertes Interesse notwendig ist, um ein Fach zu wählen, das in der Schule überhaupt nicht gelehrt wird, während es viel näher liegt, aus Unentschiedenheit oder Unsicherheit eben das Fach zu studieren, in dem man während der Schulzeit am besten war. Unspezifisches Interesse und an fremden Beurteilungen festgemachte Motivationen betonen aber den Tauschwertcharakter von Wissen im universitären Sozialverkehr und machen so für die Notwendigkeit des Bluffs zugänglicher.

Der zweite Faktor, die inhaltliche Spezifik der Fächer in ihrer Wirkung auf die Studenten stellt sich vor allem im unterschiedlichen Verhältnis zum Stoff dar: In einem Fach wie Jura, Physik oder Hochbau ist der größte Teil des Stoffes, den der Student während seines Studiums erarbeitet, kodifiziertes, klassifizie rbares und unbestreitbares Wissen, das man hat oder nicht, das also sehr schwer durch Bluff vorgetäuscht werden kann. Nicht der Zwang zum Bluff ist also das Problem, sondern die Belastungen durch das überreglementierte und überfrachtete Studium. 
Ganz anders in den Geistes- und Sozialwissenschaften: ,... es fehlt nicht nur an der äußeren Organisation des Studiums, sondern deren Mängel sind oft Ausdruck höchst divergierender wissenschaftstheoretischer und methodischer Auffassungen der für die Lehre verantwortlichen Wissenschaftler 24)".

Die Auseinandersetzung zwischen den unterschiedlichen wissenschaftstheoretischen, methodischen und politischen Richtungen erfolgt vor allem sprachlich argumentativ - schriftlich in den wissenschaftlichen Zeitschriften, mündlich in den Lehrveranstaltungen. Selbst die Kontrolle darüber, ob die eigene Arbeit richtige und sinnvolle Ergebnisse gebracht hat, kann nicht durch konkrete, überpriffbare Handlungen vollzogen werden, wie dies etwa in experimentellen Fächern der Fall ist. In den Geistes- und Sozialwissenschaften, für die man sich vor allem durch Bücher lesen und Nachvoliziehen fremder Gedankengänge ausbildet, kann man die Relevanz und Richtigkeit dessen, was man sich angeeignet hat, nur dadurch überprüfen, daß man das Gelernte in der wissenschaftlichen Diskussion in eigenen Worten fur den Diskussionszusammenhang formuliert. Hat man etwas nicht kapiert - und das kann sich erst in dieser wissenschaftlichen Kommunikation herausstellen, die zugleich hierarchiebildende soziale Kommunikation ist - dann hat man eben nicht bloß Korrektur erfahren, die erst weiteres Lernen ermöglicht, sondern man scheint zugleich blamiert. Die Folge ist der Zwang, so vorsichtig und taktisch zu formulieren, $\mathrm{d} a ß$ man sich von allgemeinen, vagen Formulierungen, von denen man sich jederzeit distanzieren kann, langsam an präzisere und inhaltlich festgelegtere Aussagen herantastet, dabei ständig auf die Reaktionen - besonders den Gesichtsausdruck der anderen achtet, um sich beim ersten bedenklichen Augenbrauenzucken wieder in unkompromittierende Unverbindlichkeit retten zu können 25).

Jede Kommunikation wird so zu einer Prüfung, über die diese besondere Spielart des Bluffs zwar immer wieder weghilft; die Unsicherheit, Entfremdung und Einsamkeit werden aber weiter verstärkt.

Unsere Erklärung für die erhöhte politische Aktivität und Konfliktanfälligkeit der Studenten der Gesellschafts- und Geisteswissenschaften geht also davon aus, daß in diesen besonderen Studienrichtungen das Leiden unter der Institution Universität besonders akut ist, weil die dort stärker mögliche und notwendige Verhaltensform des Bluffs besonders isolierend ist, und $\mathrm{daß}$ danm eine Bewegung, die die Notwendigkeit des Bluffs und damit das Leiden unter der Universität reduziert, indem sie sich in anti-autoritärer Weise politisch gegen das herrschende System richtet, in diesen Fächern eine besonders breite spontan-identische Unterstützung finden muß. Ist diese Identifikation einmal erfolgt, so kommt in diesen Fächern noch verstärkend die größere Nähe der fachspezifisch geforderten Inhalte zu den Theorien der Studentenrevolte hinzu.

24) Goldschmidt, D.: Die objektive Studicnsituation ..., a. a. O., S. 59.

25) Die weite Verbreitung der Redewendungen, mit denen sich der Red ner von dem, waser sagen will schon distanziert, bcvor er überhaupt angefangen hat, sind Indiz für diesen Zwang. Beispiele: Ich würde sagen wollen, dals... Einmal irs Unreine formuliert, würde ich meinen ... etc. 


\section{Differenzierung nach Schichtenzugehörigkeit}

Aus dem oben gesagten geht hervor, daß zum Studium in den Geistes- und sozialwissenschaftlichen Fächern vor allem sprachliche Fähigkeiten notwendig sind - der eloquentere Student, der flüssig und überzeugend sprechen oder aber wenigstens schriftlich feinsinnig differenzierend formulieren kann, hat einen Vorsprung gegenüber anderen. Nun ist bekanntlich das Verhältnis zur akademisch distanzierenden und analytischen Sprache ganz entscheidend durch die Schichtenzugehörigkeit geprägt. Die schichtenspezifischen Sprachbarrieren haben bereits eine stark selektive Wirkung während der Schulzeit, die aber nicht nur die niedrige Zahl von Arbeiterkindern an der Universität als ganzer - verglichen mit dem Anteil der Arbeiter an der Gesamtbevölkerung - bewirkt, sondern zusätzlich noch über den Erfolg in den Schulfächern die wenigen Arbeiterkinder von den stark sprachlich orientierten Sozial- und Geisteswissenschaften wegkanalisiert 26).

Die wenigen Studenten proletarischer Herkunft, die trotz allem in dieser Studienrichtung zu finden sind, werden es daher besonders schwer haben. (Die aus sprachlichen Schwierigkeiten resultierende soziale Isolierung ist im übrigen auch ein wesentlicher Grund für die außerordentliche hohe Konfliktanfälligkeit bei Ausländern) 27).

\section{Geschlechtsspezifische Differenzierungen}

Nach allen vorliegenden Daten wirkt sich die Institution Universität am verheerendsten auf Frauen aus: Die Rate von Suiziden und Suizidversuchen ist bei ihnen besonders hoch und weicht viel stärker von derjenigen der Frauengesamtpopulation der gleichen Altersgruppe ab als das bei Männern der Fall ist. Sie kommen häufiger in die psychotherapeutischen Beratungsstellen und brechen um ein Vielfaches häu figer ihr Studium erfolglos ab (dabei ist meist das angegebene Motiv, die Heirat, eher willkommener Anlaß als begründetes Motiv 28).

Der Grund für diese Häufung ist zu suchen in der spezifisch weiblichen Sozialisation für ihre Rolle im gesellschaftlichen Reproduktionsprozeß. Da den

26) Vgl. Holder, R. L.: Sex, Social Class and Student Performance, in: Universities Quarterly. London, Vol. 24, 1970, N. 2, P. 166-172.

27) Vgl. Lungershausen: Selbstmorde ... a. a. O., S. 22 und S. 26: Die Rate liegt bei ihnen um $18 \%$ bei Suiziden und um $46 \%$ bei Versuchen höher als bei einheimischen Studenten. Dabei gibt es keine nationale Differenzierungen. Vgl. auch: Jahnkes, S. und Ziolko, H.U.: ,Untersuchungen an ausländischen Studenten bei neurotischen Störungen", in: Psychische Störungen ... a. a. O., S. 245-256.

28) Vgl. Lungershausen: Selbstmorde ... a. a. O., S. 25 und 58. Die deutsche Universität ist darüberhinaus besonders frauenfeindlich. Das zeigt die Untersuchung von Anger, Hans: Probleme der deutschen Unversität. Bericht über eine Erhebung unter Professoren und Dozenten, Tübingen 1960: 1960 lag der Prozentsatz weiblicher Universitätslehrer im Gebiet der BRD mit 3,5\% (Planmäßige Professoren sogar nur 0,6\%) bei einem Anteil von 21,4\% an der Gesamtzahl der Studierenden sogar unter den Prozentanteilen von Spanien. Noch schlimmer sind die verbalen Äußerungen der befragten Professoren zu diesem Problem, z. B. unterstellten $34 \%$ Studentinnen andere Studienmotive als Studenten, wobei das ,Angeln" eines standesgemäßßen Ehemannes im Vordergrund stand (Anger S. 451 (461): Wenn sie nett sind, werden sie geheiratet, der Rest ist negative Auslese (483). 
Frauen die Aufzucht der nächsten Generation weitgehend allein überlassen ist, müssen bei ihnen auch die dafür notwendigen Einstellungen und Verhaltensdispositionen besonders gefördert werden: Libidinöse Zuwendung, Einfühlungsvermögen, geduldige, abwartende Selbstdarstellung etc.; während Aggressivität, emotionale Distanzierung, aktive Konfliktfähigkeit und andere für die Auseinandersetzung an der Universität förderliche Verhaltensdispositionen als, unweiblich ${ }^{6}$ unterdrückt werden 29).

Die durch die Notwendigkeit des Bluffs besonders libidofeindliche und isolierende Atmosphäre der Universität muß sich daher so schon viel gravierender auf die stärker libido-orientiert erzogenen Frauen auswirken. Dazu kommt aber noch - ein von allen Seiten abstrakt erhobener - bei den linken Studentinnen auch noch verinnerlichter Emanzipationsanspruch, der zu einer Distanzierung von der eigenen, $z$ war anerzogenen, aber doch vorhandenen Geschlechtsrolle auffordert 30), der also zu den übersteigerten intellektuellen Ansprüchen auch noch übersteigerte Verhaitensforderungen stellt. Diese Überforderung drückt sich oft in einem Hinund Herschwanken zwischen zwei extremen Rollen aus: Einerseits der Rückgriff auf die anerzogene Weiblichkeitsrolle, die wenigstens ein Minimum von emotionaler Sicherheit bietet, die aber auch ihre besonderen Leistungsanforderungen stellt, wie Schönheit und Nettigkeit, und andererseits - besonders dann, wenn jemand auf diese Rolle anspricht - als Negation: Offene intellektuelle Aggression. Es ist nur zu verständlich,wenn sich bei Frauen an der Universität häufig eine allgemeine Unsicherheit und emotionale Isolierung mit extremen Selbstwertzweifeln zum Dauerzustand entwickelt.

\section{E. Die Situation der Studenten im Grundstudium}

Die folgende Darstellung basiert außer auf den in Fußnoten aufgeführten empirischen Untersuchungen auf eigenen Erfahrungen aus dem Bereich der Geistesund Sozialwissenschaften und kann daher nur mit den oben entwickelten Differenzierungen auf andere Fächer und Bereiche übertragen werden.

Der Wechsel von der Schule an die Universität bedeutet eine ungeheure Verunsicherung 31): Die Schule mit ihrem engen Zusammeెnhang in der Schulklasse

29) Vgl. Müller, Ludmilla: Die Nichtbewertung der Arbeit der Kinderaufzucht im Kapitalismus und ihre Folgen für das Bewußtsein der Frauen. Diplomarbeit Soziologie, Berlin April 1972, vervielfältigtes Manuskript, S. $66 \mathrm{ff}$.

30) „Hier macht sich der entscheidende Mangel der sozialistischen Emanzipationstheorie bemerkbar, nämlich das Fehlen psychologischer Kategorien, das sie bei sozialen und politischen Postulaten stehenbleiben ließ, die von den Frauen eine Assimilation an die Männer erforderte und ihre Geschlechtlichkeit verleugnete", Bölke, G.: Die Wandlung der Frauenemanzipationstheorie von Marx bis zur Rätebewegung; Hamburg 1971, S.21.

31) „Tatsache ist, dal3 der heutige Studienanfänger sich bei der lmmatrikulation einer neuen Welt konfrontiert sieht, die, falls er nicht selbst aus akademischem Milieu stamnt, erhebliche Orientierungsprobleme aufgibt. Die Jagd nach Zulassungsgelegenheiten, Einschreiblisten, auf Sitzplätze in überfüllten Auditorien ete, das Wählen zwischen Pflichtvorlesungen und fakultativen Lehrveranstaltungen bedeutet für den cinstigen Abiturienten cine Umstellung seiner Arbeitsnethodik, die sich grundegend von der rezeptiv einord nenden Schülerhaltung unterscheidet." (W. Böker, a. a. O., S. 144) 
und den bekannten, vorausschaubaren $\mathbb{Z}$ wängen, den abgestuften Lernschritten, die an den Schüler herangetragen werden, denen er sich nur zu stellen braucht; diese Situation wird abgelöst durch eine Situation, die selbst unter den Bedingungen der technokratischen Hochschulreform noch gekennzeichnet ist, durch Anonymität, Undurchschaubarkeit und Desorganisiertheit: Man kennt keinen, keiner weiß richtig Bescheid, niemand ist zuständig, keiner hat den Überblick daruiber, was die vielen verschiedenen Stellen verlangen. Der Anfang ist in der Regel nicht als Anfang eines vierjährigen Lemprozesses gekennzeichnet, sondern wird von den Erstsemestern selbst als erste Vorbereitung auf die Abschlußprüfung verstanden. (Dies kommt bei der Eingangsstudienberatung zum Ausdruck, wenn die Frage nach den Examensbedingungen ständig wiederkehrt; und tritt als Karikatur hervor, wenn bei einem Unfall ein Student, der im 2. Semester Medizin studiert, mit dem Hinweis zur Hilfe aufgefordert wird, er sei doch Mediziner).

Haben die Übungen erst einmal angefangen, so wirkt die abstrakte Forderung, nicht bloß Anfänger, sondern Politologe zu sein, allein dadurch, daß einige wenige sie erfüllen wollen oder zu erfüllen scheinen. Dieser für Erstsemester übersteigerte Anspruch wird noch durch die Gebrauchswertseite des Wissens unterstützt, denn in der Behandlung eines jeden Teilproblems wird der Student über diesen Teil hinausgewiesen auf den gesellschaftlichen Gesamtzusammenhang und dazu noch auf Berge von Literatur, die er meint eigentlich lesen zu müssen. In einem nach Semesterjahrgängen gegliederten Studium wird wenigstens verhindert, daß in ein und derselben Übung Examenssemester mit Erstsemestern zusammensitzen, andererseits kann derjenige, der es nicht schafft, sich an den Diskussionen zu beteiligen, sich nicht mehr vor sich selbst damit entschuldigen, daß die Diskussionsteilnehmer höhere Semester sind. Das, was in den Erstsemesterübungen, also an echten oder scheinbaren Leistungen erbracht wird, wirkt nun voll als von jedem $\mathrm{zu}$ erfüllender

Hier muß kurz auf den Versuch eingegangen werden, das zu allen Zeiten tendenziell gesellschaftlich unangepaßte Verhalten von Studenten auf ein bloß entwicklungspsychologisches Problem zu reduzieren, indem die studentische Situation als ,verspätete Adoleszenz" (E. H. Erikson und H. Reichenbacher, referiert bei: W. Böker: Ebda, S. 142), „festgehaltene Kindersituation" (J. Krüber:,Die sozialen Bestimmungsgründe der studentischen Universitätskritik", in: Psychische Störungen a. a. O., S. 32) oder einfach als „Pubertät ${ }^{\text {"6 }}$ definiert wird. Z. B.: ,,... sollte man bei den erschwerten Anpassungsproblemen an die Forderung des Studiums nie den vordergründigen soziologischen Aspekt der Pubertät außer acht lassen. Diese Entwicklungsperiode kann erst dann als abgeschlossen angesehen werden, wenn der junge Mensch sich auf der Ebene des angestrebten Berufes selbst erhalten kann. ${ }^{66}$ (D. Langen:, Studentische Probleme aus der Sicht prolongierter Pubertätsproblematik, in: Psychische Störungen . . . a. a. O., S. 148). Demnach wäre das Kriterium der vollen charakterlichen Reife, ob man in den Arbeitsprozeß - wo möglich, in den Kapitalverwertungsprozeß integriert ist oder nicht. Die ernst zu nehmende entwicklungspsychologischen Überlegungen zu diesem Problem finden $\mathrm{m}$. E. ihre reale Basis in den von D. Goldschmidt: Die objektive Studiensituation... S. 53, und Böker (ebda, S. 145 f.) sowie M. L. Moeller für die Prüfungssituation beobachteten Erscheinungen, die von den genannten Autoren damit erklärt werden, dałs durch die Institution Universität frühkindliche Ängste reaktiviert werden, die in dem für ,Extravaganzen ${ }^{6}$ toleranten universitärem Raum freier ausgelebt werden können als im kapitalistischen Arbeitsprozeß. (M.L.Moeller: „Zur Psychodynamik der neurotischen Prüfungsangst", in: Psychische Störungen ... a. a. O., S. 221). 
Anspruch.

Da die Studenten als Schüler darauf erzogen worden sind, ihr Wissen dem Lehrer zur Begutachtung und zur Benotung vorzulegen, setzt sich diese Verhaltensweise an der Uni fort. Das hat zur Folge, daß diejenigen Studenten, die die wirklichen oder projezierten Wissensansprüche wirklich oder per Bluff erfüllen können, sich in keiner Weise didaktisch zu ihren Kommilitonen verhalten. Ihr Ziel ist nicht, sich verständlich zu machen, sondern ihr Wissen zu produzieren, darzustellen. Dieses Verhalten verstärkt nun die oben skizzierten, institutionellen $\mathbb{Z}$ wänge der Universität, und die scheinbare oder wirkliche Erfülung der übersteigerten Anspriche wirkt durchaus terroristisch auf die anderen Teilnehmer der Übung, ohne daß dies so intendiert wäre. So bildet sich schon während der ersten Wochen eine Hierarchie heraus, die unter den übersteigerten, verinnerlichten Ansprüchen dazu erzieht, sich die notwendigen Blufftechniken anzueignen, denn ohne sie scheint man in Gefahr, herauszufallen. Man muß Zusammenhänge auch dort glatt formulieren können, wo man sie selbst noch nicht sieht.

Wer noch nicht genügend Selbstvertrauen zum glatten Formulieren hat, der steht in Gefahr, verkürzt zu formulieren, nicht verstanden zu werden - niemand geht auf den Beitrag ein, er ist aus dem Diskussionszusammenhang herausgefallen. Geschieht dies öfter, dann hört man bei bestimmten Beiträgen schon gar nicht mehr richtig zu. Häufen sich solche Mißerfolge, so kann in dieser Phase des Studiums noch per Fächerwechsel oder Abbruch des Studiums ausgewichen werden. Im Hauptstudium stellt sich das Problem dann gravierender (dem entspricht die höhere Inzidenz von Suiziden und neurotischen Störungen im Hauptstudium).

Da nur wenige die übersteigerten Ansprüche erfüllen können, oder eine so hohe Frustrationstoleranz haben, zu warten, bis sie sich über die Gebrauchswertseite das nötige Wissen erarbeitet haben, da also die meisten darauf angewiesen sind, sich ihren Platz in der Sozialhierarchie und ihr Selbstwertgefiihl über die Tauschwertseite des Wissens zu erkämpfen, sind nahezu alle mehr oder weniger darauf angewiesen, sich während der ersten Semester die nötigen Blufftechniken anzueignen. Diese sind vor allem sprachliche Techniken. Stellt man die richtigen Worte (Verwertungsschwierigkeiten, reale Bewegung des Kapitals, Theorie und Praxis, Dialektik, Emanzipation, Pluralismus etc.) in den richtigen verbalen Zusammenhang, so kann das wirken, als ob man den gesellschaftlichen Zusammenhang selbst richtig begriffen habe. Allerdings ist es ein schwieriges und gefährliches Unterfangen: Es ist wie wenn man auf das frische Eis hinausgeht, von dem man noch nicht richtig weiß, ob es hält: Man setzt den Fuß nu r zaghaft auf und verlagert das Gewicht ganz sachte und langsam auf die neue Stelle und wenn es knackt, zieht man sich schnell zu rück. Und auch wenn es eine Weile nicht geknackt hat, man muß sich doch ganz leicht machen, man kann eigentlich gar nichts machen, was Spaß macht, man muß immer darauf achten, nur sachte aufzutreten, denn man weiß nicht, ob das Eis trägt. Und was das Schlimmste ist: Auf dünnem Eis kann man nicht zusammenstehen, da muß man Distanz halten. So wie die Bluffsprache verunsichert, isoliert und aktionsunfähig macht, so macht sie auch lernunfàig. Da die Blufftechnik darauf zielt, Fehler zu vermeiden, Mängel zuzudecken, werden Hypothesen und Fragen tendenziell nicht mehr formuliert, wenn sie das Risiko des 
Fehlerhaften und Unvollständigen in sich tragen. Es sind aber genau diese ,dummen Fragen, ohne die Lernen gar nicht möglich ist.

Darüberhinaus steht der Benutzer der Blufftechniken in der Gefahr, in folgende Zwickmühle zu geraten: Gelingt ihm die Beherrschung der Blufftechniken nicht, so sorgt die Häufung von Mißerfolgserlebnissen daf̂ur, daß der behandelte Stoff negativ besetzt und damit die an der Gebrauchswertseite des Wissens orientierte Motivation ungeheuer erschwert wird. Gelingt aber die Beherrschung der Blufftechniken, so ist zwar das Nachholen der Gebrauchswertseite möglich, aber die Wahrscheinlichkeit ist groß, daß sich nur neue Anspruchsebenen eröffnen und es nicht mehr nötig erscheint, den beschwerlichen Umweg über das feste Ufer zu machen.

Der Druck, solche Techniken zu erlernen, ist heute bei politisierten Erstsemestern besonders stark. Zum einen ist der Umkreis des sozial geforderten Wissens bei Marxisten besonders groß und der Einstieg in das in sich gegliederte System gesellschaftlicher Zusammenhänge besonders schwierig, weil das richtige Einzelne ohne den Begriff des Ganzen falsch wird. Zum anderen wirkt der Anspruch, in der täglichen Agitation, in der politischen Arbeit und in der Auseinandersetzung mit anderen politischen Richtungen argumentativ überlegen zu sein, die Einsicht in die gegliederte Totalität also schon zu haben. Aus dieser Notwendigkeit entspringt die Tendenz, den Marxismus als bloßes Instrument zu begreifen, mit dem man „Bürger knacken“" kann, und die Abneigung, sich mit bürgerlicher Wissenschaft konkret-inhaltlich auseinanderzusetzen, sie auf die reale Basis der Ideologie hin zu untersuchen, sie als wichtigen Teil der bürgerlichen Wirklichkeit anzusehen, und sich durch sie verunsichern zu lassen.

Unter der Herrschaft dieser überwältigenden Zwänge zum Bluff taucht immer wieder die Gefahr auf, daß sich Begriffe zu bloßen Formulierungen und Schlagwörtern verselbständigen, die von Lippe zu Lippe springen und so in einer Art osmotischem Prozeß zur ,herrschenden Lehre` werden, ohne je auf breiter Basis selbständig erarbeitet und begriffen worden zu sein.

Bei den durchaus inhaltlich politisierten und bewußten Genossen ist dieses bloße Erlernen einer Sprache, die die Teilhabe an der Totalität der realen Klassenkämpfe und ihrer Theorie signalisiert, ein Weg, der durch die Institution Universität beinahe aufgedrängt wird, um die lange Zeit zu überbrücken, bis die Gebrauchswertseite des Wissens nachgeholt ist. Und genau hier liegt dann die Gefahr, daß aus der osmotisch entstandenen herrschenden Lehre ein Dogmatismus der stimmigen Formulierung wird und vor lauter kämpferischer täglicher Praxis die theoretische Durchdringung der realen Bewegung nicht mehr geleistet werden kann. (Eine Organisation mit formalisierter Hierarchie, in der der einzelne Student Aufträge ausführt, die von oben aus dem größeren Zusammenhang kommen, auf den er in der Agitation und Auseinandersetzung hinweisen kann, ist in dieser Situation von besonderer Attraktivität. Die anderen, loseren Organisationen, die im Lernprozeß immer wieder schwanken und ihre Begrenztheit und Beschränktheit zugeben, sind dagegen in ihrer Wirkungsweise auf junge Semester nur noch mit den Seminaren früherer Jahr zu vergleichen: Langjährige Genossen und hohe Semester fechten in ,hochtheoretischen“" Auseinandersetzungen um die Bestimmung der 
richtigen Linie und appellieren an die jungen Genossen, Stellung zu beziehen, die erarbeitete Linie nach außen zu vertreten, ohne den Lernprozeß der jungen Genossen in Stufen zu organisieren).

Das Grundstudium wird also durch die Institution Universität zu der Periode, in der die universitäre Tauschwertseite des Wissens sich durchsetzt. Das äußert sich besonders deutlich darin, daß die Berufsorientienung, die bei den meisten Erstsemestern als Ausdruck der konkreten Nützlichkeit des zu erlernenden Wissens explizit vorhanden ist, in den folgenden Semestern bei vielen Studenten verloren geht 32), und durch die Ansammlung von Wissen um der Ansammlung selbst willen, also durch die innenuniversitäre Tauschwertseite des Wissens ersetzt wird. Oft drängt sich die Frage der Benufsorientierung erst wieder als unbeantwortete Frage nach dem bestandenen Abschlußexamen in den Vordergrund. Meist aber und verschärft durch die angespannte Arbeitsmarktlage für bestimmte Gruppen von Intellektuellen und die politischen Einstellungskontrollen schlägt die Berufsorientierung auf die Gestaltung des Hauptstudiums durch. Das Hauptstudium steht also unter der Gefahr, entweder dieses Problem zu verdrängen oder aber die Berufspraxis nur per Anpassungsdruck wahrzunehmen.

\section{F. Die Situation der Studenten im Hauptstudium}

Die Lage der Studenten im Hauptstudium wird hauptsächlich durch das drohende Abschlußexamen bestimmt. Zwar kann sich in der ersten Zeit des Hauptstudiums trotz der institutionellen Zwänge nach den jahrelangen Studien eine dominant inhaltliche orientierte Motivation entwickelt haben, die auf diesem Gebiet den Bluff als störende und lemfeindliche Verfälschung zurückweist und klares, selbstbewußtes Arbeiten ermöglicht. Diese Möglichkeit, ein selbstbewußtes, gebrauchswertorientiertes Verhältnis zur Wissenschaft und zur Institution Universität $\mathrm{zu}$ entwickeln, ist je nach individueller Sozialisation unterschiedlich ausgeprägt.

Dieser Möglichkeit wirkt aber in allen Fällen die Abschlußprüfung als übermächtige Tendenz entgegen: sie hebt - wie oben entwickelt - vor allem auf die Tauschwertseite des Wissens ab, zwingt zur Gleichgültigkeit gegenüber dem konkreten Inhalt und stellt damit die strukturelle Analogie zwischen Wissen und Ware wieder voll her. Dies um so mehr, da die meisten Studenten ein völlig unrealistisches Verhältnis zu den Prüfungen haben: 1. weil die Prüfungsordnungen mit ihren Forderungskatalogen eine Enzyklopädik des Faches aufstellen, die von niemandem in einem zeitlich begrenzten Studium erfüllt werden kann und die dem wirklichen Prüfungsverlauf als im allgemeinen wohlwollende Diskussion um einige

32) Vg1. Mielitz, Reinhard: ,Die Situation der Studienanfänger in der Philosophischen Fakultät - dargestellt am Beispiel der Philosophischen Fakultät Freiburg i. B. " in: Neue Sammlung, Göttingen, Viertes Sonderheft 1967.

Der Grund für diesen Wandel ist wohl darin zu suchen, daß sich das Denken und Verhalten immer mehr auf die universitären Bezugsgruppen und ihren intellektuellen Sozialnormen ausrichtet. Dadurch wird eine Tendenz zur Verselbständigung der Intellektuellen-Welt von jeder Realität erzeugt, die man in dem Theaterkommunismus mancher politischer Hochschulgruppen durchaus wiedererkennen kann. 
vorbereitete Spezialgebiete tatsächlich nicht entspricht; 2. weil vielfältige psychische Konflikte sich an der Prüfungssituation aktualisieren können, so daß der reale Anlaß in den Hintergnund tritt und auf die Prifungen Anforderungen projiziert werden, die sie zu einer mythischen Bewährungsprobe für alle Facetten der Persönlichkeit verselbständigen 33).

So erzeugt die Prufung mit all ihren wirklichen, offiziell vorgespiegelten und unkontiollierbar projizierten Anforderungen einerseits den gewaltigen $\mathbb{Z}$ wang, sich in den Bluff zu fliichten, der so oft scheinbar geholfen hat; andererseits bedeutet die Prüfung aber gerade die Drohung, das der Bluff durchschaut, die vorgeschützte Tauschwertsumme auf ihren reaien Gebrauchswertinhalt, die Kreditwirdigkeit uberprüt wird. Die Stoffe, die Gegenstand der drohenden Prüfung sind, werden durch die Angst negativ besetzt. Zugleich wird die inhaltiche Konzentration auf subjektiv interessante Stoffe zerstreut, um die möglicherweise geforderte Breite im Wissen zu erreichen. Die bereits bestehenden Minderwertigkeitsgefühle und Unsicherheiten erzeugen das widersprüchliche Bedürfnis, den Bluff immer perfekter zu gestalten, wodurch die Angst vor der Aufdeckung in der Prüfung und damit die affektiv negative Besetzung des Stoffes noch weiter zugespitzt wird; und schließlich - wenn sich keine Guppe zusammenfindet, die sich gegen die Prüfung gemeinsam wehrt und so wieder eine Basis für Solidarität und Selbstvertrauen aufkommen läßt - kann sich Prüfungsangst und negative Besetzung des Prüfungsstoffes bis zur völligen psychischen Blockade und selbstmörderischem Fluchtverhalten aufschau-

33) „Nicht die mangelnde intellektuelle Leistungsfähigkeit ist die primäre Ursache der Prüfungsangst, sondern das unbewußte Erleben der Prüfungssituation als eine nicht verarbeitete Gefahrensituation der Kindheit in Zusammenhang mit Triebkonflikten". $M$. L. Moeller: ${ }_{33}$ Zur psychodynamik der neurotischen Prüfungsangst ${ }^{a b}$ in: Psychsiche Störungen a. a. O., \$. 221.

Dem kommt auch der irrationale Aufbau der meisên Prüfungen entgegen, der mit den unvorbereitbaren Klausuren und zeitlich rigide beschränkten Hausarbeiten mehr einem Hindernisrennen als einer wissenschaftlichen Leistung gleicht und damit seinen Ursprung in den mythischen Initiationsriten fruherer Gesellschaftsstufen erkennen läß3t.

Der so kïnstlich gesteigerte Stress wirkt sich durchaus unterschiedlich auf unterschied. ich disponierte Personen aus und stellt so eine Diskriminielung her: ,Stress ... hat bei den betroffenen Personen mehr Leistungsmotivation angeregt, in vielen Fällen aber Furcht, Selbstschutz - und Aggressionstendenzen gefördert und hat Selbstzweifel und Selbstunterschätzung bis hin zu Straferwartungen und Regressionstendenzen erzeugt. Die emotionale Reagibilität wird eingeengt, man ermüdet leichter.

Im intellektuellen Bereich können unter Stress Auffassungsschärfe und Reaktionsgeschwindigkeit abnehmen, ebenso wie die Leistungsgüte und oit auch die Leistungsmenge; stereotypes, rigideres Denken tritt an die Stelle schöpferischen, flexibleren Denkens, das zur Entnahme von Anregungen aus der Umwelt befähigt.

Diese Ergebnisse gelten in besonderem Małse für milberfolgsängstlichc Personen; bei Erfolgszuversichtlichen können durch Stress u. U. erst die letzten Reserven zu einer positiven Leistung mobilisiert werden. " (I. Wagner: ${ }_{99}$ Ubber den Einfluß von Situationsfaktoren in Leistungsprüfungen ${ }^{k s}$ in: Psych ische Störungen ... a. a. O., S. 177)

$\mathrm{Zu}$ den bereits genannten Faktoren komrnt noch oft eine Trennungsangst, die sich mit dem Prüfungsereignis verbindet, weil es das Ende eines Lebens unter wenigstens vertrauten Bedingungen bedeutet und in neue bedrohende Ungewílsheit stößst. Dazu eindrückliche Fallgeschichten bei: Böker, a. a. O., S. 145 ff.. 
keln 34). ,Geklagt wird z. B. über Verstimmbarkeit und Erschöpfbarkeit bei Konzentrationsaufgaben: über Kopfschmerz, Schwindel und Schweißausbruch bei der Lektüre von Lehrbüchern, über Unrast, Merkfähigkeitseinbuße, Lustlosigkeit, allgemeine Mattigkeit und Schlafbeeinträchtigung. Man köntie es allein auf der Bude nicht mehr aushalten, die Decke stürze ein, man brauche Menschen um sich oder Radiomusik.

Andere spüren keinen Antrieb mehr, erwachen morgens bleischwer, bleiben lange liegen und ziehen sich am Abend bald wieder mit schlechtem Gewissen ins Bett zurück, weil sie den Tag hindurch nichts hinter sich bringen konnten. Die Zukunft bedrückt sie wie ein Berg.

Häufig ist das Gefihl der Sinnlosigkeit, der allgemeinen tiefen Skepsis über den Zweck und die Verwendungsmöglichkeiten des aufgespeicherten Wissensstoffes, dessen gesellschaftliche Nutzanwendung dunkel blieb. Der Einstieg in komplexere Fachprobleme gelingt dann immer schwerer und unwilliger, ...".35)

\section{G. Die Situation der Dozenten}

Zwar wirkt der reale und projizierte Wissensdruck in den Übungen und im Kollegengespräch auf die Dozenten noch viel stärker als jemals auf die Studenten, aber bei allen Dozenten ist wenigstens ein Spezialgebiet vorhanden, auf das sie sich als Basis ihres Selbstbewußtseins immer wieder zurïckziehen können und zudem haben sie die Blufftechniken bis zur Perfektion erlemt und geübt. Viel schlimmer als der Wissensdruck ist daher der Originalitätsdruck d.h. der $\mathbb{Z}$ wang, die eigene Qualifikation durch neue, möglichst epochemachende Problemlösungen unter Beweis zu stellen. Dabei ist der Originalitätszwang zugleich Veröffentlichungszwang, da die formale Qualifikation durch Veröffentlichungen eingelöst werden muß 36).

Ist die neue Problemlösung keine epochemachende Entdeckung, die in ihrem Gebrauchswert von allen Fachwissenschaftlern anerkannt werden muB, so lassen sich auf die Veröffentlichungen als Ausweise der Qualifikation bei Bewerbungen und Berufungen völlig verselbständigte Tauschwertkriterien anwenden: Oft wird der

34) C. Bloch (,,Y-a-t-il une psychopathologie de l'étudiant?" in: Psychische Störungen... a. a. O., S. 17) nennt die Zah1 von $33 \%$ der Studenten, die über, ,inhibitons intellectuelle" klagten. Böker berichtet, dafs $85 \%$ der Studenten, die die Beratungsstelle aufsuchten über Lern- und Arbeitsstörungen klagten (Böker, S. 139) und resümiert, daßs schwere Arbeitsstörungen vor dem. Examen das häufigste Symptom war (S. 150).

Franz Baumeyer (,Arbeitsstörungen bei Studenten" in: Zeitschrift für psychosomatische Medizin, Göttingen, J. 14, 1968, H. 2, s. 82) stellt die Hypothese auf, daß sich die allgemeinen Symptome erst im Hauptstudium so verschärfen, dals in den schlimmsten Fällen der Arzt aufgesuch t werden mus und zwar meist erst der praktische Arzt, weil die Symptome somatische Ausprägungen erhalten. Diese Entwicklung mag auch Erklärung dafír sein, dał die meisten Selbstmorde von Studenten verübt werden, die 6. und höhere Semester sind. (Lungerhau sen, Selbstmorde ... a. a. O., S. 37).

35) Böker, S. 140

36) Nur als Indikator: die Pol. Dok. - eine Dokumentation über wissenschaftiche Zeitschriftenaufsätze aus dem Bereich der Politologie - hat in den letzten sieben Jahren allein aus diesem Bereich über 12000 Aufsätze besprochen. 
persönliche Eindruck, der aus Vorurteilen, Sympathien, Abneigungen oder politischen Differenzen stammen mag, im nachhinein durch ein Urteil über die „Qualifikation“" gestützt, indem die Zahl der Veröffentlichungen, die Anzahl der Fußnoten, Zitate und Literaturverweise verglichen und auf ihre formale Richtigkeit überprüft wird, um so durch die völlige Abstraktion von der konkret-qualitativen Seite der Wissenschaft aus den Bewerbern um Stellen und Titel den „Qualifiziertesten" auszuwählen. Bekanntlich geschieht dies besonders deutlich gegenüber marxistischen Bewerbern, was so eklatante Fälle wie in jüngster Zeit der Fall Holz und die soziale Medizin in West-Berlin dokumentieren 37).

Aber auch bei nicht-marxistischen Dozenten wirkt diese irrationale Form der Selbstrekrutierung der Hochschullehrerhierarchie ausgesprochen terroristisch: Entweder man unterwirft sich völlig einem „Förderer" und gibt damit alle eigenständigen Ansätze auf, die dem ${ }_{n}$ Förderer" gegen den Strich gehen könnten, oder man muß alles daran setzen, eine wirklich epochemachende Entdeckung zú publizieren - eine Zwangssituation, wie man sie sich ungünstiger für inhaltlich orientiertes Arbeiten kaum vorstellen kann 38).

Da die Originalität Ausschließlichkeit für ihren Inhalt beansprucht, muß sie sich aus dem Individuum schöpfen. Der Originalitätsdruck wirkt daher isolierend und gegen die für die inhaltliche Arbeit wichtige Kommunikation und Diskussion. Da dies sich zum verschärften Wissensdruck addiert, besteht auch auf der Dozentenebene eine Tendenz zur Versachlichung der Beziehungen, zur Distanzierung und zur Unterd rückung der Libido.

Diese Distanzierung ist begleitet von einer institutionell verstärkten Hierarchisierung. Die an der Spitze der Hierarchie Stehenden müssen nicht mehr um ihre Position kämpfen, sie sind sich oft nicht einmal mehr der Tatsache bewußt, daß sie eine Position in einer Sozialhierarchie innehaben. Sie brauchen ihre Position me ist nicht einmal zu verteidigen, weil sie eben aufgrund ihrer Spitzenposition so viel Selbstbewußtsein haben können, daß sie sich voll auf die Gebrauchswertseite des Wissens verlegen können: Sie können es sich manchmal leisten, mal nicht Bescheid zu wissen, die ,dummen ${ }^{6}$ Fragen zu stellen, geduldig, großzügig und selbstkritisch zu sein und dadurch immer besser und mehr zu lernen und so ihre Position zu festigen, ohne es selbst zu bemerken.

Diese selbstsichere und gebrauchswert-orientierte Haltung ist jedoch sehr selten. Normalerweise sind alle Dozenten, ob Assistenten, Professoren oder Lehrstuhlinhaber unter permanentem Konkurrenzdruck und müssen sich daher

37) Erst im Fall Mandel und bei dem Problem der Einstellung von Studenten mit ausgezeichneten Abgangszeugnissen in den Staatsdienst, bei denen sich nun überhaupt nichts finden lassen konnte, was man auf der Ebene der, Qualifikation hätte vorbringen können, bei diesen Fällen wurde dann per Senat bzw. Ministerpräsidentenerlał offen politisch argumentiert.

38) Vgl. dazu insbesondere: Untersuchungen zur Lage der deutschen Hochschullehrer, 3 Bände, hrsg.: K. Plessner, Göttingen 1956 und dort im ersten Band: Ders.: Zur Soziologie der modernen Forschung und ihrer Organisation in der deutschen Universität - Tradition und Ideologie, 1924, ebda, S. 19 - 36, insbes. S. 31 ff., sowie im gleichen Band: Dietrich Goldschmidt: Die gegenwärtige Problematik (1956), S. 37-49. Die Abschaffung des ,Privatdozenten ${ }^{\circ}$ hat an dieser Situation nichts wesentliches geändert. 
verstärkt nach der Tauschwertseite des Wissens orientieren - und je weiter oben sie in der Hierarchie stehen, wenn sie gar nach Weltruhm streben, desto eitler, verletzlicher und terroristischer müssen sie in der Weitergabe des verinnerlichten Druckes und in der Ausbeutung ihrer Umgebung sein.

Die vielen, die unten und in der Mitte der Hierarchie sitzen und weiter hinauf wollen oder wenigstens nicht absinken wollen, stehen in der Gefahr, sich voneinander $z u$ isolieren, ihre Beziehungen auf das sachlich Notwendige zu reduzieren. Sie müssen empfindlich reagieren auf alles, was ihre Position, deren sie sich gelegenüich schmerzlich bewußt sind, zu verschlechtern droht, und zugleich müssen sie so tun - und das erst recht, wenn sie links sind - als ob sie eben dies nicht täten, woraus nur neue Empfindlichkeiten entstehen. So hat die Tauschwertseite des Wissens auf Dozentenebene einen besonders isolierenden und sprengenden Charakter wegen dem Zwang zur individuellen, schöpferischen Originalität.

Es ist aber nịcht nur der Originalitätsdruck, der auf Tutoren und Assistenten Konkurrenzdruck ausibt, sondern es kommt gegen Vertragsende noch der Flaschenhals der Stellen hinzu: es gibt sehr viel weniger Stellen für Promovierte als für Nichtpromovierte; und die Promovierten müssen sich dann wiederum um die wenigen Professorenstellen schlagen. Dieser institutionalisierte Auslesemechanismus heizt natürlich auch den $\mathbb{Z}$ wang zur Orginalität weiter an, daneben aber entsteht ein extremer Anpassungsdruck an die Personen und Kriterien, die über den Aufstieg entscheiden. Je dickbäuchiger die Flasche und je enger der Flaschenhals, desto schleimiger und intriganter droht die Konkurrenz zwischen den zukünftigen Bewerbem um die wenigen Stellen zu werden. Die Tauschwertseite droht absolute Dominanz zu erhalten.

Dem wirkt aber der auf Dozentenebene viel unmittelbarere und unausweichlichere Zwang zur Gebrauchswertseite des Wissens entgegen - und dieses Gegengewicht wiegt bei marxistischen Dozenten, die an der bürgerlichen Universität forschen und lehren noch viel schwerer: Die konkret-inhaltlichen Anforderungen der Gestaltung und Aufrechterhaltung von Lehre und Forschung, die Auseinandersetzung mit bürgerlicher Wissenschaft und bürgerlichen Wissenschaftlern wirken so unmittelbar als Gebrauchswertseite des Wissens und als die praktische Notwendigkeit des organisatorischen Zusammenschlusses, daß es über alle institutionell erzeugte Isolienung und Distanzienung zu einer ständigen Kooperation im organisatorischen und $\mathrm{zu}$ ständigen gegenseitigen Hilfen im inhaltlichen, wissenschaftlichen Bereich kommen kann 39). Dies ist aber nur der eine Aspekt der Faktoren, die für die Lage insbesondere der marxistischen Dozenten bestimmend sind. Denn die institutionellen $\mathbb{Z} w a ̈ n g e$ der Universität wirken zuerst einmal äuße rlich weiterhin über den Zwang zur formalen Weiterqualifikation über Dissertation und Habilitation (auf Hochschullehrerebene wird sich die amerikanische Situation des ,publish or perish " auch immer mehr in Deutschland durchsetzen). Da die Formalqualifika-

39) Ein Versuch in diese Richtung stellt die SAZ (Sozialistische Assistenten Zelle) am Fachbereich 15 (Otto-Suhr-Institut) der FU Berlin dar. Sie wurde 1969 in der Phase gegründet, als sich die Studenten aus der Hochschulpolitik für etwa 2 Semster zuriickzogen. 
tionen an einer burgerlichen Universitat erworben werden müssen und da fur alle Prüfungen gilt, dak nur die Antwort richtig ist, die der Prüfer für richtig hält, schlagen die burgerichen Wissenschaftskriterien schon auf institutioneller Ebene in jede auch noch so solidarische Dozentengruppe durch: Der Originalitätsdruck macht nach den Themen der anderen schielen.

Weil marxistische Wissenschafiler sich aber nicht so einfach willkürlich ein Thema wählen kônen, sondern die zusammenhängende methodische Entfaltung des Gegenstandes bestimmte Fragen in bestimmter Reihenfolge aufwirft und bearbeitbar macht tiffit der institutionelle $Z$ wang zur individuellen Originalitä bei Marxisten auf eine Gruppe von Leuten, die meist zu gleicher Zeit über sehr eng verwandte Fragen arbeiten, de daher ganz besonders stark auf dauernde gegenseitige Information fit dic inhathiche Weiterfihrung angewiesen sind, die aber umso mehr furchten mussen, dak die Weitergabe von Informationen und Entdeckungen zum Scheitem an der Hürde der formalen Qualifikation führt.

Zu diesem äußeren Druck kommt nun als extrem verschärfender Faktor hinzu, daß der Originalitätsdruck bei den Dozenten, ähnlich wie der Wissensdruck bei den Studenten weniger auf realen institutionellen Anforderungen beruht, als auf den Projektionen der sozialen Anforderungen, die in den hierarchisch strukturierten Gruppen erzeugt und hochgetrieben werden. Diese sozialen Anforderungen haben gerade bei marxistischen Wissenschaftlern eine Tendenz, zum Alpdruck anzuwachsen. Da es sich alles, was geschrieben wird, gefallen lassen muß, an der „methodischen Elle" Marx gemessen zu werden, kann man schließlich nichts mehr schreiben als Verrisse anderer Arbeiten, indem man feststellt, daß sie der ,,methodischen Elle" nicht genügen. Die Liste dariber, was alles getan, berücksichtigt, zuerst aufgearbeitet und miteinbezogen werden „müßte " wird so umfangreich, daß man gar nicht mehr anfangen kann, weil jeder Anfang mit der Falschheit des Vorläu figen, Partikularen und eben Unvollkommenen behaftet ist. Man kommt von der methodischen Ebene der Betrach tung des allgemeinen Kapitalbegriffes gar nicht mehr herunter und ist in der inhaltlichen Weiterentwicklung völlig blockiert.

Diesem doppelten Druck kann man sich einige Zeit entziehen, indem man auf Hochschulpolitik oder andere allgemein für wichtig erachtete Tätigkeiten ausweicht, ein Weg, der sich bei der gegenwärtigen Lage an den Hochschulen und der Terminüberlastung während des Semesters, die wissenschaftliche Forschung nahezu unmöglich machen, geradezu aufdrängt. Dies um so mehr, da eine aktive Hochschulpolitik Bedingung der Möglichkeit marxistischer Lehre und Forschung an der bürgerlichen Hochschule ist, da sie sonst von der bürgerlichen Wissenschaft als "dogmatisch" und a priori unwissenschaftlich einfach aus dem Bereich sozial akzeptablen Wissens und damit auch aus der Institution Universität wegdefiniert würde.

\section{H. Das Verhältnis zwischen Bozenten und Studenten}

Auch heute noch treten die Dozenten in den Übungen im allgemeinen weder didaktisch geschult, noch mit didaktischen Intentionen auf, sondern entweder werden Paukveranstaltungen des Grundstudiums abgehalten oder die Studenten werden im Hauptstudium fur die Z wecke der Dozenten instrumentalisiert, d. h. der 
Dozent setzt Studenten auf die Problematik seine: jeweiligen Arbeit an und fordert Problemiösungen auf seinem Problemlösungsiveau 40). Durch eine solche, völlig undidaktische instrumentalisierung wird der instibutionelle Druck auf die Studenten noch verschärf, die Lemsituation erschwert. Das Wissen des Dozenten und seine assoziativen Randbemerkungen oder seine Insistenz auf die Totalität umfassende Systematik, Gie standig auf noch zu Erarbeitendes hinweisen, erzeugen tendenziell einen solchen Druck, daf die Studenten inn in irgendeiner Weise reduzieren oder abwehren müssen, wenn es nicht gelingt, zu einer durch die Gebrauchswertseite des Wissens bestimmte Zusammenarbeit zu gelangen.

Dabei gibt es zwei Wege: Der eine ist der Weg der defensiven Arroganz, der übrigens auch in jeder ancieren Hierarchie zur Anwencung kommt; in Gesprächen über den Dozenten wird er mit allen Mitteh abqualifiziert, um so den Druck zu reduzieren. Da diese Kritk aber meist nur von einer Ebene aus möglich ist, die ein noch höheren Anspruchsniveau darstellt als dasjenige, gegen das man sich durch Kritik wehren will, wird durch den Abwehrversuch selbst der Druck noch verschärft. Das ist̂ das Schimme an der defensiven Arroganz, daß sie sich ständig selbst einen noch gewichtigeren Grund schafft, einen neuen isolierenden Affront zu produzieren und so die Unsicherheit noch zu verschlimmern. (Dies gilt selbstverständlich auch für das Verhältnis zwischen den Dozenter - für Marxisten auch nur dann nicht, wenn sie sich bewukê dagegen wehren).

Zwischen linken Studenten und linken Dozenten nimmt dieser Weg der Anspruchsreduzierung meist nicht den Verlauf defensiver Arroganz, sondern besteht konkret im Vorwurf des Seminarmarxismus, der selbst dann erhoben wird, wenn derjenige, der den Voiwurf enhebt, selbst kein anderes Praxisfeld als das der Hochschule hat. Dabei geht einerseits der Begriff davon, was nun wirklich den Seminarmarxismus ausmacht, nämîch die bloß geistesgeschichtliche Beschäftigung mit Marx, veiloren und andererseits wird damn nur zu leicht die praktische Relevanz einer Theoriebildung übersehen, die es sich zum Ziel setzt, die Kritik der Politischen Okonomie für heute zu rekonstriieren also die reale Bewegung zu analysieren und so zu einer Strategie-Bestimmung beizutragen.

Dieser Vorwurf wird durch eine strukturelle Differenz in der Stellung zur Hochschulpolitik bestärkt: Die Dozenten haben selbst als Tutoreñ mit einer Vertragsdauer von 5 Jahren (mit den Verlängenngen) eine längerfristige Perspektive in der aktiven Gestaltung der Hochschulpolitik als die Studenten. Thre Politik wird daher tendenziell auf länge re Dauer und geduldigere Entwicklung angelegt sein. Sie wird daher in allgemeinen weniger radikal sein und damit den Vorwurf auf sich ziehen, bloß ständische Interessenvertretung zu sein - ein Vorwurf, der durch die permanente Wiederholung seinen Sinn,zur selbstkritischen Überlegung anzuhalten, völlig verliert (auch dann, wenn sie wirklich nötig wäre). Sie ist auch darum weniger radikal, weil sie auf Dozentenebene nie vordergründige Mobilisienungspolitik oder primär Propagandafeldzug, sondern eben bloße Sicherungspolitik für die Möglich-

40) Vgl. D. Goldschmidt: Die objektive Studiensituation..., a. a. O., S. 59 und für den amerikanischen Bereich: D. R. Brown: Student Stress and the Institutional Environment, Nr. 3, S. 92-107, insbesondere S. 98 f. 
keit marxistischer Forschung und Lehre ist (und an der bürgerlichen Universität als vom industriellen Kapitalverwertungsprozeß relativ abgehobene staatliche Institution auch nur sein kann).

Der zweite Weg besteht darin, da wo eine unüberwindbare Distanz gegeben ist, den Druck, den diese Distanz ausübt, dadurch abzuwehren, daß die Distanz offen als unüberwindbar deklariert wird. Den total Überlegenen begegnet man mit jüngerhafter Verehrung. Den Uneingeweihten gegenüber verhält man sich dann gern als Eingeweinter.

In beiden Fäilen ist Solidarität oder gar eine libidinös besetzte Beziehung zwischen Dozenten und Studenten nur gegen den universitären Zusammenhang möglich, denn wie immer die Abwehr erfolgt, sie wirkt auf Dozenten wie Studenten verunsichernd und isolierend. Besonders im Fall der defensiven Arroganz reagiert der angegriffene Dozent tendenziell mit immer perfekterer, dichterer und unangreifbarerer Argumentation, die Lernprozesse noch mehr behindert und das Verhältnis von Druck und Gegendruck noch weiter eskaliert.

\section{Die Situation der ,anderen Dienstkräfte“}

Nach dem Berliner Universitätsgesetz werden diejenigen Universitätsmitglieder, die weder Studenten noch Dozenten sind, , andere Dienstkräfte' genannt. Soweit sie mit den Studenten und Dozenten überhaupt nicht direkt in Kontakt kommen, unterscheidet sich ihre Situation nicht wesentlich von der anderer staatlicher Bediensteter in gleicher Funktion (Rechnungs-, Bibliotheks- und Archivwesen, Verwaltung etc.). Für diejenigen unter ihnen, die einen Hochschulabschluß haben, gilt allerdings genauso wie für akademisch gebildete ,andere Dienstkräfte' überhaupt, daß sie vor sich selbst wie vor anderen, immer mit den Dozenten verglichen werden und in Versuchung sind, zu meinen, sie müßten ihren Status ,rechtfertigen".

Diejenigen ,anderen Dienstkräfte', die dauernd mit Studenten und Dozenten umgehen müssen, haben darunter zu leiden, daß sie ganz unten auf der universitären Sozialhierarchie angesiedelt sind. Denn als Personen, die an der Konkurrenz um wissenschaftliche Stellen, Prüfungsnoten und intellektuell-wissenschaftliche Anerkennung institutionell nicht beteiligt sind, haben sie auch keinen Einfluß darauf, was man wissen muß - oder besser: wovon man auf keinen Fall merken lassen darf; daß man es nicht weiß -, um akkzeptiert zu werden. Man kann sie also ohne Nachteil nicht beachten oder arrogant behandeln, scheint es. Sie gehören somit gar nicht zu der Bezugsgruppe, die das allgemeine Klima der Universität bestimmt, sondern werden vielmehr meist als bloßes Hilfsmittel behandelt, um die universitären Funktionen aufrecht zu erhalten. Entsprechend gestalten sich dann auch die sozialen Beziehungen zwischen Studenten und Dozenten einerseits und den ,anderen Dienstkräften ${ }^{6}$ andererseits: Die Dozenten - egal ob links oder nicht - soweit sie auf die Arbeitsergebnisse der, anderen Dienstkräfte angewiesen sind, tendieren zu einem Verhalten, das dem eines betont freundlichen und zuvorkommenden Arbeitgebers gleicht, der sein treues Personal nicht verlieren will - eine Attitüde, die dadurch nur noch penetranter wird, daß versucht wird, die Stimmen der ,anderen Dienstkräfte ${ }^{6}$ in den Gremien für die eigene Position in den 
hochschulpolitischen Auseinandersetzungen zu instrumentalisieren. Das Ergebnis ist eine Atmosphäre süßlich-klebriger Intrige statt der Vertretung klarer und eigenständiger Interessen.

Die Studenten, die meist weniger auf die Arbeit der , anderen Dienstkräfte angewiesen sind - oder dies wenigstens meinen können - gestalten ihre sozialen Beziehungen zu den Pförtnern, Sekretärinnen, Bibliothekarinnen, Mensabediensteten etc. je nach individueller Erziehung und aufgestauter Aggression unterschiedich. Allgemein ist jedoch die Tendenz, daß sie den , anderen Dienstkräften' gegenüber, weil diese über keine hierarchie-relevanten Sanktionen verfügen, viel leichter, weil folgenloser, Aggressionen abführen können, sei es durch distanzierte Arroganz oder bloße Gedankenlosigkeit oder schlichte Unverschämtheit. Diese Tendenz gilt selbstverständlich auch für linke Studenten, insbesondere da sie - wie gezeigt - dem Wissensdruck besonders extrem ausgesetzt sind. Bei ihnen besteht aber der wichtige Unterschied, daß ihr Verhalten besonders kritisch beobachtet wird - erst recht dann, wenn ,links' sein als moralische Anstrengung, es besser zu machen als die anderen' interpretiert wird (ein Mißverständnis, das leider von einigen studentischen Organisationen ständig gefördert wird). Das Resultat ist eine meist mißtrauische bis feindselige Einstellung der , ande ren Dienstkrä fte ' gegen die Studenten (besonders gegen die, linken') - also gerade bei der Gruppe von Werktätigen, denen die Studenten täglich begegnen.

\section{Schlußbemerkung}

Eigentlich gehört an den Schluß eines solchen Aufsatzes die Antwort auf die Frage "Was folgt daraus?". Dazu bin ich aber weder fähig noch willens, denn das würde eine genaue Kenntnis der Verhältnisse an der jeweiligen Universität und der Geschichte und Struktur der einzelnen politischen Gruppen voraussetzen.

Einige Hinweise kann man jedoch auch ganz allgemein geben: 1. Solange die Universität eine von der Produktionssphäre relativ isolierte Intelektuellenklave ist, lassen sich die skizzierten Zwänge und die Tendenz zur realitätsblinden Verselbständigung von Theoriegespinsten auch auf politischer Ebene nur bekämpfen, aber nicht völlig abschaffen.

2. In allen Fällen wird es das entscheidende Mittel zur Bekämpfung dieser Zwänge sein, sie nicht als individuelles Versagen vor dem moralischen Anspruch zu brandmarken, sondern sie immer wieder am eigenen Verhalten aufzuzeigen und bewußt zu machen als produziert durch die kapitalistische Form der gesellschaftlichen Reproduktion. Dadurch kann es vielleicht gelingen, einmal die Auswirkungen dieser $Z$ wänge in der eigenen Gruppe zu reduzieren und zugleich ein weitverbreitetes Bedürfnis unter den Universitätsmitgliedern als politisches Bedürfnis anzusprechen. Beides ist entscheidend, um die grundsätzliche Solidarität wiederherzustellen, die wichtigste Bedingung einer antikapitalistischen Perspektive ist, die aber auch nirgendwo in der Form bedroht ist wie bei Intellcktuellen. 